\title{
The identity of the jihadist and the psychosocial mechanisms of adhesion to the terrorist
}

\author{
L'identità del jihadista e i meccanismi psicosociali di adesione \\ alla causa terroristica
}

\section{La identidad del yihadista y los mecanismos psicosociales de adhesión a la causa terrorista}

\author{
Lorenzo Ortenzi \\ Dottore in Investigazione, criminalità e sicurezza internazionale, Università degli Studi Internazionali di Roma \\ UNINT; Dottore in Scienze sociali e organizzative, Università Cattolica del Sacro Cuore, Italy
}

\begin{abstract}
The elaboration of effective strategies to fight terrorism, such as the Islamist one, must be based on a careful analysis of the phenomenon and of the main actor, the jihadist. Scientific research has given discordant and not fully favorable results towards the acceptance of the hypothesis of a psychopathological component as a cause of radicalization. Rather, the cause must be sought in a multiplicity of factors such as individual, social, political and religious ones. Radicalization, also favored by the wide use of cyberspace by terrorist organizations, therefore plays a role of "resocialization" for all those vulnerable subjects, affected by a deep existential crisis.
\end{abstract}

\section{RIASSUNTO}

L'elaborazione di efficaci strategie di contrasto ad un terrorismo come quello di matrice islamista non può non passare per un'attenta analisi del fenomeno e dell'attore protagonista, il jihadista. La ricerca scientifica ha dato esiti discordanti e non pienamente favorevoli verso l'accoglimento dell'ipotesi di una componente psicopatologica come causa di radicalizzazione. Piuttosto, la causa va ricercata in una molteplicità di fattori come quelli individuali, sociali, politici e religiosi. La radicalizzazione, favorita anche dall'ampio utilizzo del cyberspazio da parte delle organizzazioni terroristiche, gioca quindi un ruolo di "risocializzazione" per tutti quei soggetti vulnerabili, colpiti da una profonda crisi esistenziale.

\section{RESUMEN}

La elaboración de eficaces estrategias de lucha al terrorismo como el de matriz islámica, necesita un análisis minucioso del fenómeno y de su actor protagonista, el yihadista. La investigación científica ha producido resultados divergentes y no muy favorables hacia la aceptación del hipótesis de una componente psicopatológica como causa de radicalización. Más bien, la causa se tiene que buscar en una multiplicidad de factores como los individuales, sociales, políticos, religiosos. La radicalización, también favorecida por el largo uso del ciberespacio por parte de las organizaciones terroristas, juega un rol de "re-socialización" para todos sujetos vulnerables, que se ven afectados por una profunda crisis existencial.

\section{Il concetto di terrorista}

Prima di giungere alla trattazione dell'identità del jihadista, occorre innanzitutto comprendere cos'è o chi è un terrorista.

Cercare di rispondere a domande relative alla definizione di terrorista risulta tanto complesso quanto il cercare di dare una definizione di terrorismo. Parte della difficoltà risiede nella grandissima mole di documenti accademici sul terrorismo, la quale può rendere ardua la valutazione e la misurazione della completezza della conoscenza sull'argomento. Il problema dell'identificazione del terrorista potrebbe inoltre anche essere ricondotto alle opere di isolamento da parte degli studiosi dei soggetti che si stanno studiando. Concentrarsi infatti esclusivamente sulla definizione di terrorista e adottare un approccio esclusivamente psicologico al terrorismo significa prendere in considerazione soltanto un atto- re, distogliendo l'attenzione dal gruppo sociale, dal quale il soggetto proviene e trae legittimità per il comportamento terroristico. Non è possibile, pertanto, separare il terrorista dalla società, in quanto fortemente radicato in essa. La società rappresenta il contesto ambientale che lo genera, lo supporta, lo dirige, lo controlla e che inevitabilmente influenza il suo agire (Horgan, 2015).

Molto importante nello studio del terrorismo e nell'elaborazione di efficaci strategie di prevenzione e contrasto risulta l'interpretazione delle motivazioni, degli stimoli e dei percorsi di vita dei terroristi (Picozzi, 2016). Questo approccio in stile weberiano permette l'individuazione dei fattori soggettivi che spingono un individuo ad abbracciare la causa terroristica e rappresenta uno dei cardini fondamentali per efficaci operazioni di intelligence proattiva. In questo contesto osservare personalità che presentano determinate caratteristiche, analizzare gli eventi che interessano l'individuo e le reazioni emotive derivanti possono consentire di 
comprendere la psicologia dell'uomo e le sue pulsioni nascoste (Teti, 2018)

In generale, il terrorista si distingue dagli altri criminali a causa di tre fattori, ovvero l'uso della violenza, la clandestinità e il fine politico. La violenza, considerata illegittima e pericolosa, distingue le azioni terroristiche dal ricorso legittimo della forza da parte delle forze dell'ordine, in quanto i terroristi vengono a meno del diritto internazionale legato alla guerra. I terroristi, per la natura stessa delle loro condotte criminali, hanno bisogno di mimetizzarsi all'interno della società che vogliono colpire, vivono nell'ombra e tendono a nascondersi per poi agire. La clandestinità, quindi, differenzia il terrorismo dalle altre forme di violenza politica. Da ultimo, per quanto riguarda il fine, la criminalità comune e quella organizzata si distinguono dal terrorismo in quanto mosse da finalità di tipo economico e di lucro. L'obiettivo del terrorista è infatti di natura politica e ideologica e il reato diventa un mezzo per raggiungere lo scopo, ovvero la destabilizzazione politica e sociale (De Pasquali, 2016).

Da un punto di vista criminologico, il terrorista si distingue dal cosiddetto "mass murder", ovvero l'assassino di massa. Per mass murder si intende un assassino che uccide diverse persone, contestualmente e nello stesso luogo. Le vittime coinvolte sono per lo più persone sconosciute all'aggressore e prese casualmente. Apparentemente, una strage del genere potrebbe essere erroneamente classificata come di matrice terroristica. Tuttavia, per convenzione, le stragi realizzate da mass murder non comprendono quelle di tipo terroristico, in quanto un'azione terroristica è incoraggiata da motivi estrinseci e non da motivazioni intrinseche dello stragista, tipicamente non razionali. Pertanto, quando una strage presenta una motivazione riscontrabile nel reale e non solo nella psiche del soggetto, questa non è classificabile come strage effettuata da assassini di massa (Mastronardi \& Leo, 2005). Volendo esemplificare, Mohamed Bouhlel, trentunenne tunisino che si è scagliato con un camion sulla folla radunatasi lungo la Promenade a Nizza il 14 luglio 2016 durante le celebrazioni della Presa della Bastiglia non può essere considerato un assassino di massa. Bouhlel, infatti, nonostante fosse noto per la sua instabilità mentale e pur non facendo parte dello Stato Islamico, era stato persuaso e si ispirava al suo messaggio, pertanto la motivazione di quell'attacco è da ricondurre a fattori esterni alla psiche individuale. Sarebbe erroneo, infatti, così come per la maggior parte dei terroristi, ricondurre le sue azioni a meri problemi mentali, trascurando il fattore ideologico (Vidino, Marone \& Entenmann, 2017). Inoltre, da un punto di vista operativo, l'attentatore tunisino ha utilizzato un veicolo come arma mentre, come sottolineano Mastronardi \& Leo (2005), il mass murder predilige principalmente armi da fuoco.

È bene inoltre distinguere il terrorismo dai cosiddetti "hate crimes", i crimini dell'odio. Quest'ultimi, che intendono colpire e punire direttamente i membri di specifici gruppi sociali, basandosi sul pregiudizio e sulla discriminazione, sono generalmente commessi d'impulso, non sono rivendicati e pubblicizzati e non implicano la presenza di gruppi aventi un programma politico o un orientamento ideologico relativamente chiaro (Vidino, Marone \& Entenmann, 2017).

Il terrorista è definito come un soggetto che aderisce ad una dottrina o ad una causa che finisce per sopravvalutare. Il terrorista, nell'ambito del processo di radicalizzazione, diviene inizialmente vittima di un indottrinamento o lavaggio del cervello, per poi deumanizzare le sue vittime, considerate esseri inferiori e disprezzate. Queste vengono considerate come oggetti di cui il terrorista può decidere la sopravvivenza o la morte. Il terrorista si considera quindi un essere superiore che si batte per una giusta causa e che vede nel terrore l'unico mezzo per realizzare i propri fini (Mastronardi \& Leo, 2005).
Ciò che è stata per molti decenni, ed è tuttora, al centro dei dibattiti di sociologi e psicologi è la questione riguardante la causa che spinge una persona a uccidere e mutilare deliberatamente un'altra persona. Per alcuni esperti si tratta di individui psicopatici affetti da gravi psicopatologie. Altri, invece, ritengono i terroristi soggetti mentalmente deboli e che possiedono un incontenibile livello di aggressività (Teti, 2018). Per lungo tempo gli studi dei ricercatori si sono focalizzati sulla psicologia degli attentatori, suggerendo l'esistenza di particolari sottotipi di personalità, tra cui spiccano la paranoia e il narcisismo, i tratti più comunemente associati ai terroristi. Per di più, risulta semplice creare un collegamento tra psicopatia e terrorismo, in quanto i terroristi, così come gli assassini psicopatici, presentano spesso assenza di rimorsi e di senso di colpa per le loro azioni. I terroristi sono infatti soliti trovare delle giustificazioni al loro comportamento, sottraendosi alle proprie responsabilità. Tuttavia, la tesi della psicopatia è ancora limitata e vi sono scarse prove secondo cui i terroristi possano o debbano essere considerati degli psicopatici. Inoltre, l'egocentrismo patologico presente nelle psicopatie sarebbe in contrasto con le caratteristiche che i reclutatori cercano in un individuo da reclutare nell'organizzazione terroristica, ovvero elevato impegno, disciplina, affidabilità e resistenza allo stress (Horgan, 2015).

Studi come quello di Martha Crenshaw, docente della Stanford University, si discostano dalle tesi positiviste e rilevano, al contrario, come ciò che accomuna i terroristi è la normalità, ovvero la conduzione di una vita ordinaria priva di qualsiasi psicopatologia. La causa dell'affiliazione al terrorismo va quindi ricercata in altri fattori, come l'infanzia vissuta, il contesto familiare, il livello di istruzione, le dinamiche sociali e tutte quelle esperienze che hanno contraddistinto, positivamente o negativamente, la propria esistenza (Teti, 2018).

Horgan (2015), seguendo la stessa linea, continua affermando che la tendenza a concentrarsi esclusivamente sulla drammaticità dell'evento porta inevitabilmente a spiegare il comportamento focalizzandoci sulla persona che ne è responsabile, ritenendola in qualche modo "diversa" e "anormale". In questo caso si è vittima di quello che in psicologia sociale viene chiamato "errore di attribuzione", ricavando una visione distorta del terrorista e, più in generale, del terrorismo stesso. L'errore di attribuzione o bias di corrispondenza è un errore inferenziale molto comune ed è definito come "la tendenza sistematica attribuzionale generale che porta le persone a considerare in modo eccessivo il comportamento come il prodotto di stabili caratteristiche di base della personalità" (Hogg \& Vaughan, 2012, pag. 49). Questo errore si manifesta quando le persone tendono a concentrarsi automaticamente sul soggetto anziché sul contesto, divenendo più saliente nell'elaborazione dell'informazione. Nei casi più estremi, l'errore di attribuzione può assumere la problematica forma dell'essenzialismo, per cui "le persone non solo attribuiscono le cause del comportamento a disposizioni di fondo, ma considerano tali disposizioni come caratteristiche immutabili e spesso innate dell'individuo o del gruppo a cui appartiene" (Hogg \& Vaughan, 2012, pag. 50). Horgan (2015) ritiene che questo comportamento possa essere considerato come una sorta di meccanismo di difesa della società, che si sente in questo modo più sicura e in grado di comprendere un gesto estremo come un attacco terroristico. Si ritiene utile, piuttosto, una focalizzazione sui leader del terrorismo e sui processi di gruppo che svolgono un ruolo essenziale nei processi di reclutamento e nella coesione del gruppo stesso. Tuttavia, nonostante le ricerche abbiano presentato evidenze sulla "normalità" del terrorista, questa tesi non è così diffusa nella letteratura, la quale presenta tuttora analisi che si rifanno ad una anormalità psicologica.

Il terrorista, da un punto di vista generale, è, quindi, un individuo arrogante, sicuro di sé, ma sospettoso nei confronti degli altri. 
Egli è una persona che aderisce ad una dottrina che finisce per supervalutare, è calcolatrice, priva di rimorso, paranoide, fredda nei riguardi delle vittime, litigiosa e completamente assorbita nell'ideologia del proprio gruppo a causa dell'intenso processo di indottrinamento o brainwashing. Il terrorista, inoltre, è dotato di un alto quoziente intellettivo e di un'altrettanta elevata capacità di disumanizzazione. Per quanto riguarda le caratteristiche demografiche, queste possono variare in base ai diversi gruppi terroristici ma generalmente i terroristi sono soggetti giovani, perlopiù uomini, con un tasso di istruzione medio-basso e una condizione economica difficile. Si tratta inoltre di individui che sono venuti a contatto con gruppi violenti o armi e che fanno del concetto di violenza uno stile di vita (Palermo \& Mastronardi, 2005).

\section{Il fondamentalismo islamico}

L'estremizzazione della religione islamica, ovvero il fondamentalismo islamico, gioca un ruolo essenziale nel "nuovo terrorismo" e nel processo di adesione alla causa terroristica. Infatti, il fondamentalismo religioso, assieme alla presenza di terroristi suicidi, è l'elemento che contraddistingue il terrorismo islamista dalle altre forme di violenza terroristica che avevano nelle ultime decadi del XX secolo diffuso il terrore in tutta Europa (De Pasquali, 2016).

Il fondamentalismo islamico può essere definito come un atteggiamento convinto nei confronti di un preesistente valore, di un fondamentale, di un punto di vista o di una regola formulata che non possono essere posti in discussione. Il fondamentalismo presenta sempre un nesso inscindibile con la tradizione e può essere di natura religiosa o secolare. Una religione fondamentalista si basa quindi su principi e verità di fede che sono preesistenti e indiscutibili (De Luca, Macrì \& Zoli, 2013). Lombardi (2016) propone una fondamentale distinzione, quella tra radicalismo e terrorismo, in quanto essi sottintendono concetti complessi e diversi tra loro, intorno ai quali non esiste una perfetta convergenza teorica, normativa e interpretativa. Esistono infatti due prospettive a confronto: la prima individua un ideale continuum tra i due termini in cui il terrorismo è l'apicale e violenta manifestazione; la seconda li ritiene ontologicamente differenti, in quanto il radicalismo non implica necessariamente l'utilizzo della violenza, a differenza del terrorismo.

A partire dagli anni Ottanta il mondo ha assistito ad un intensificarsi dell'escalation di azioni terroristiche legate al fondamentalismo religioso, dovuto dall'inasprirsi ed al radicalizzarsi di alcuni focolai di conflitto già esistenti, soprattutto in Medio Oriente e in Africa settentrionale (De Pasquali, 2016).

La prima esperienza dell'Europa con il terrorismo di matrice jihadista, per come lo conosciamo oggi, risale al 1995, quando una rete strettamente connessa all'Algeria porta a termine una serie di attacchi in Francia, come rappresaglia per il coinvolgimento di Parigi nella guerra civile algerina. Due anni prima una cella di militanti attiva tra New York e il New Jersey, sotto la guida spirituale dello "Sceicco cieco" Omar Abdel-Rahman, fa esplodere un ordigno nel garage del World Trade Center, provocando la morte di sei persone e il ferimento di oltre mille (Vidino, Marone \& Entenmann, 2017).

Il primo esempio di gruppo radicale islamico è stato quello dei Fratelli Musulmani, nato in Egitto nel 1929, che inizialmente si è occupato di insegnamento e altri impegni di tipo civile. Con lo scoppio della guerra arabo-israeliana del 1967, che ha portato alla creazione all'interno del mondo musulmano di gruppi estremisti dediti ad azioni clandestine, e con la questione palestinese, il fondamentalismo islamico ha subito un'ulteriore radicalizzazione. Avvenimenti come la rivoluzione sciita in Iran nel 1979 per mano di Khomeini, la guerra in Libano, l'uccisione del presidente egiziano Sadat e la guerra del Golfo degli anni Novanta hanno alimentato l'integralismo islamico e favorito il connubio tra terrorismo e fondamentalismo islamico, il quale aveva oramai cominciato a prendere atto dei propri fallimenti politici (Mastronardi \& Leo, 2005).

Tuttavia, è con al-Qaeda e Bin Laden che il fondamentalismo islamico ha raggiunto la massima espressione e manifestazione, quest'ultima da individuarsi nell'attacco dell'11 settembre 2001. Inoltre, questa corrente radicale ha continuato a sopravvivere, seppur in forma diversa, anche dopo lo smantellamento di al-Qaeda e delle sue basi in Afghanistan, attraverso i terroristi palestinesi, nell'eterna diatriba contro gli israeliani, e i terroristi ceceni, per il riconoscimento della loro autonomia da Mosca. Ciò nonostante, sebbene si tenda ad associare il fondamentalismo di matrice islamista alle più recenti manifestazioni terroristiche, quali al-Qaeda e Stato Islamico, questo fenomeno ha origini ben più antiche. In particolare, esso nasce nell'Ottocento nell'ambito del dibattito interno alla comunità musulmana per ciò che concerne la necessità di opere di modernizzazione e soltanto successivamente ha acquisito quell'accezione di violenza che possiede oggi. In questo contesto, molti studiosi fanno risalire la progressiva decadenza del mondo musulmano alle caratteristiche dell'Islam stesso, riferendosi proprio alle sue correnti più radicali. Questa visione, condivisa dai paesi occidentali e dalle classi sociali arabe più elevate, ritiene che il declino arabo e musulmano sia riconducibile alla mancata realizzazione di riforme sociali, politiche e culturali necessarie e all'esistenza di dettami incompatibili con le moderne democrazie. In contrapposizione a questa visione modernizzatrice e riformista, $\mathrm{i}$ fondamentalisti islamici ritengono che le cause della decadenza siano riconducibili all'abbandono dell'integrale applicazione della legge coranica; solo ristabilendo quest'ultima sarà possibile far risorgere l'Islam, realizzare una nuova civiltà musulmana unificata e ristabilire il Califfato (De Pasquali, 2016).

La corrente del fondamentalismo islamico mira quindi ad un ritorno all'essenza del Corano, ad una sua interpretazione letterale e restrittiva, alla ricreazione della Ummah del Profeta, ovvero la comunità di tutti i musulmani istituita da Allah al di là di ogni confine spaziale, e alla proclamazione del jihād, da muovere contro i "corrotti" e "infedeli" in nome di Allah (Mastronardi \& Leo, 2005).

Il termine "jihād", o "guerra santa", descrive la lotta per acquisire la virtù e seguire la volontà di Dio e rappresenta lo sforzo di costruire una società più giusta diffondendo l'islamismo tanto con la predicazione quanto con le armi (Sinclair, 2003). In altri termini, l'obiettivo del jihād è quello di propugnare la fede musulmana tra i popoli con la persuasione, l'aggressione, la disciplina e la diplomazia, col fine di ricreare la Ummah sotto un'unica guida (Mastronardi \& Leo, 2005).

La parallela liberazione della Ummah dall'oppressione straniera, cercando l'unificazione di tutte le comunità islamiche, è senza dubbio uno dei tratti fondamentali di questa corrente in cui il nemico è spesso individuato nell'Occidente. Il sentimento antioccidentale è infatti quell'elemento comune a tutti i gruppi integralisti islamici e, quando sfocia nel terrorismo, il fondamentalismo islamico mira quindi a colpire in maniera indiscriminata i cittadini del "primo mondo", considerati espressione e simbolo dell'evoluzione degenerativa delle democrazie occidentali; gli attentati terroristici vengono così utilizzati come strumento di "purificazione" ed annientamento della cultura e della società occidentale (De Pasquali, 2016).

È tuttavia necessario evidenziare come la propaganda di matrice fondamentalista distorca la realtà sui reali conflitti nel mondo ravvisando presunte prove di uno scontro tra Islam e Occidente. Occorre ricordare in questo senso che la maggior parte della società civile musulmana, quindi la maggioranza, rifiuta l'impiego e l'istigazione alla violenza da parte dei terroristi, che rappresenta- 
no invece la minoranza. Di conseguenza, in vista di necessarie attività di prevenzione, risulta di fondamentale importanza fare in modo che la voce maggioritaria prevalga su quella dell'estremismo, modificando la percezione delle politiche nazionali ed europee (Consiglio dell'Unione Europea, 2005).

Questo sentimento di odio da parte dei fondamentalisti nei confronti dell'Occidente, accusato di essersi arricchito a danno dei paesi musulmani e di aver sfruttato le risorse, soprattutto energetiche, presenti in queste zone, è ciò che alimenta l'idea di un Islam guerriero, evoluto e potente che si vorrebbe restaurare nel presente (De Luca, 2002).

Le origini dell'odio degli integralisti verso l'Occidente sono anch'esse antiche. Per molti secoli Islam e Cristianesimo si sono contrapposti, ma l'inizio della degenerazione del rapporto tra le due religioni è da ricondurre al 1683, quando l'esercito ottomano viene sconfitto dallo schieramento formato da polacchi, austriaci e tedeschi nella battaglia di Vienna, evento decisivo della guerra austro-turca. Da quel momento in poi le armate musulmane vengono sconfitte in quasi tutti gli scontri con le truppe occidentali e nel corso dell'Ottocento il mondo islamico è stato di fatto sottomesso all'egemonia europea. Il predominio dell'Europa cristiana ha determinato un progressivo impoverimento culturale del mondo musulmano, accompagnato da una crescente debolezza e frammentazione politica e militare e da un'arretratezza economica diffusa. L'impoverimento di alcune zone e l'arricchimento di altre, in particolare di quei paesi che gestiscono la produzione del petrolio, ha creato un divario sempre più ampio nel mondo musulmano (De Pasquali, 2016).

Dalla storia di questi ultimi secoli nasce così il tentativo del mondo islamico di liberare sé stesso dall'assoggettamento europeo, facendo nascere un senso di rivolta e rivalsa nei confronti dell'Occidente. Le vittorie riportate dagli eserciti europei hanno rappresentato una pesante e indimenticabile umiliazione per il mondo musulmano, dimostrando superiorità culturale, tecnologica e militare degli occidentali (De Pasquali, 2016).

Qui, risulta fondamentale sottolineare come il passato e la storia rappresentino delle componenti sempre vive nella coscienza collettiva musulmana. Ciò è da ricollegare al diverso rapporto con la storia da parte del mondo musulmano, il quale è "teologico-politico" e non "storicistico" come per gli occidentali (Sbailò, 2014).

L'Islam rappresenta infatti per molte società contemporanee "l'ultima risorsa di senso comune" in un mondo che va trasformandosi. La motivazione può essere individuata nella sociologia funzionalista di Emile Durkheim, uno dei padri fondatori della sociologia classica, che riteneva come la religione riuscisse a costruire e mantenere in vita una coscienza collettiva ${ }^{1}$ (Pace, 2007).

Due esempi chiarificatori di questo concetto li riporta il quotidiano "La Repubblica". Il primo articolo riguarda l'attacco ai militari italiani in Iraq del novembre 2019. Nell'articolo del quotidiano romano si cita la rivendicazione dell'attacco da parte dell'ISIS attraverso un comunicato diffuso dall'agenzia di propaganda "Amaq", la quale scrive: "Con l'aiuto di Dio, soldati del Califfato hanno colpito un veicolo $4 X 4$ con a bordo esponenti della coalizione internazionale crociata ed esponenti dell'antiterrorismo peshmerga nella zona di Kifri, con un ordigno, causando la sua distruzione e ferendo quattro crociati e quattro apostati" (La

1 Nella sociologia funzionalista di Emile Durkheim, la coscienza collettiva rappresenta una sovrastruttura sociale che non coincide con le singole coscienze individuali ma in ogni modo le determina, le plasma e le orienta. La coscienza collettiva può essere definita inoltre come un sistema di credenze che storicamente può mutare ma che condiziona intere generazioni di individui (Pace, 2007).
Repubblica.it, 2019). Il secondo articolo riporta il pensiero dei fondamentalisti circa la pandemia del Covid-19, virus che ha colpito duramente i Paesi occidentali. La newsletter interna allo Stato Islamico "Al Naba" riporta come il virus sia considerato dagli islamisti come una punizione di Allah verso i Paesi idolatri, Iran sciita compreso. L'articolo incita i jihadisti a colpire nuovamente l'Occidente già provato profondamente dalla pandemia, esortando i musulmani a non avere pietà in quanto gli infedeli fanno finta di dimenticare i crimini commessi contro i musulmani e che continuano a commettere (Cadalanu, 2020).

L'utilizzo di un termine come "crociati" e i continui riferimenti ai crimini passati verso i musulmani fanno comprendere come nell'Islam la storia non sia da intendere come un insieme di fatti storici accaduti nel passato senza alcun rapporto e importanza nel presente, ma come un continuum temporale. Per l'Islam le Crociate fanno parte della "storia", ovvero del presente e della vita politica (Sbailò, 2014).

Tuttavia, non è solo l'odio e il risentimento nei confronti della cultura occidentale e delle altre religioni ad alimentare il terrorismo di matrice islamista, ma anche il continuo riacutizzarsi di conflitti di natura etnica, religiosa e politica fra i vari gruppi, presenti in Medio Oriente, in Africa e nel Mediterraneo, l'esistenza di regimi dittatoriali e corrotti, la difficoltà di creare delle moderne democrazie in questi paesi e la scarsità di risorse, soprattutto idriche e alimentari, che genera dipendenza dai paesi occidentali (De Pasquali, 2016).

Il concetto di fondamentalismo è strettamente correlato a quello di fanatismo, ovvero quell'imprescindibile fattore che guida l'agire del jihadista al di là di ogni carattere demografico, e a quello di martirismo, ossia l'impiego di "uomini-bomba" negli attentati terroristici (De Pasquali, 2016).

Il termine "fanatismo" deriva dalla religione e dal latino, in particolare dal termine "fanaticus", col significato di "tempio", il quale indicava una persona ispirata dalla divinità (De Pasquali, 2016); viene definito in psicologia da Galimberti (2006) come l'atteggiamento di chi, nell'adesione incondizionata ad una causa, perde ogni atteggiamento critico, persuadendosi dell'assoluta incontestabilità e dell'incontrovertibile legittimità di ogni atto intesa a realizzarla. Inoltre, secondo Hole (1995), il fanatismo è una convinzione personale condizionata dalla struttura della personalità, avente per oggetto contenuti e valori ridotti, caratterizzata da un alto grado di identificazione, che viene mantenuto o perseguito con fortissima intensità, persistenza e coerenza e che non è capace di scendere a compromessi con altri sistemi o altri uomini, i quali possono essere combattuti come nemici esterni, anche con l'impiego di tutti i mezzi e in conformità alla proprio coscienza.

In conclusione, sin dalla sua esplosione il terrorismo di matrice islamista ha costituito un fattore determinante a livello geopolitico, diventandone un vero e proprio attore transnazionale, non statuale, in grado di riunire le varie reti e comunità islamiche diffuse al di fuori del mondo musulmano. Esso è diventato uno dei principali fattori di destabilizzazione politica, militare, ma anche socioeconomica, rappresentando una minaccia non solo per i singoli Stati ma per l'intero scenario globale (De Pasquali, 2016).

$\mathrm{Su}$ queste basi si è sviluppato il terrorismo del XXI secolo, soprattutto in seguito agli eventi dell' 11 settembre 2001, assumendo una forma di dimensioni globali e caratterizzando quello che è il terrorismo contemporaneo, un terrorismo indiscriminato fatto di attacchi di valenza simbolica in cui lo scopo non è solo quello di mietere vittime ma anche quello di lanciare un messaggio (De Pasquali, 2016). Da questo momento in poi gli attacchi compiuti $\mathrm{da}$ attori ispirati dall'ideologia jihadista non sono più stati considerati come avvenimenti sporadici, bensì una tra le sfide cardinali alla sicurezza della maggior parte dei paesi occidentali (Vidino, Marone e Entenmann, 2017). 


\section{Nella mente del jihadista}

Avendo inquadrato da un punto di vista generale la figura del terrorista e introdotto la fondamentale componente religiosa, è ora possibile entrare nello specifico e concentrarsi esclusivamente sulla categoria del terrorista islamista, osservandone le peculiarità.

Un ingente numero di ricerche empiriche ha dimostrato che non esiste un unico profilo del militante jihadista nelle sue diverse forme. Nonostante non ci sia un unico profilo, tuttavia, è comunque possibile descrivere le popolazioni statistiche in quanto alcuni tratti individuali si presentano con maggiore frequenza nelle analisi dei profili (Marone \& Vidino, 2018).

Disegnare un profilo generalizzato del jihadista risulta un'operazione ardua se non impossibile a causa di un panorama molto vasto di tratti psicologici, sociali, motivazioni e provenienze degli attentatori. Osservando gli identikit dei responsabili degli attacchi in Europa dal 2015, si passa infatti da ragazzi immigrati di seconda e terza generazione, i cosiddetti terroristi "home made" o "home grown", con piccoli precedenti penali e non integrati a giovani appartenenti al ceto medio-borghese (Picozzi, 2016). Prende così piede il fenomeno dei "lone wolves", ovvero i lupi solitari, individui che vengono spinti alla realizzazione di un atto terroristico in nome di una scelta totalmente personale e svincolata dall'appartenenza a gruppi terroristici. Questo tipo di terrorista rappresenta una minaccia difficilmente contrastabile se non attingendo all'efficienza di quegli organi tradizionalmente preposti al contrasto della minaccia terroristica interna (Salvatori, 2018). Attentati terroristici come quello al giornale satirico francese Charlie Hebdo hanno dimostrato come i terroristi che agiscono secondo l'ideologia fondamentalista vivano ed operino indisturbati all'interno delle città europee e non necessariamente debbano colpire secondo i dogmi del terrorismo internazionale o transnazionale ed essere scovati al di fuori del paese target (De Pasquali, 2016).

Nei territori sotto il controllo dell'IS si tratta invece di foreign fighters e di appartenenti a minoranze religiose come i sunniti iracheni (Zoja, 2017). I foreign fighters sono i combattenti stranieri volontari che, a causa un forte senso di inadeguatezza, spesso determinato dalla disoccupazione o dalla difficoltà di integrarsi nella società, dopo un indottrinamento tramite Internet, abbandonano l'Europa e si recano in Siria e in Iraq per essere addestrati e per combattere tra le file dell'IS e provengono soprattutto dai paesi nordafricani e in numero più contenuto dall'Europa. Spesso sono giovani di seconda e terza generazione che individuano nel jihād uno strumento per fuggire dalla disperazione e una via per la realizzazione personale. Questi combattenti rappresentano un pericolo in quanto, una volta addestrati, possono rientrare nei paesi d'origine e compiervi attentati (Limes Online, 2015). Vidino, Marone \& Entenmann (2017) segnalano in questo senso come alcuni terroristi autori degli attentati più recenti in Europa, come quelli di Charlie Hebdo, di Parigi del 2015, di Thalys, Bruxelles \& Ansbach abbiano un passato da foreign fighters. La maggior parte di questi si era recata in Siria ed in Iraq per unirsi tra le fila dello Stato Islamico per poi rientrare nel loro paese di provenienza, alimentando il tasso relativo al cosiddetto "effetto blowback".

Questo fenomeno rappresenta in un certo senso anche un'evoluzione rispetto al terrorismo di al-Qaeda, che ha visto Bin Laden circondarsi di militanti, fiancheggiatori, veterani della resistenza afghana e militanti islamici radicali sparsi in tutto il mondo. Secondo quanto riportato da un rapporto presentato al Consiglio di Sicurezza delle Nazioni Unite dalla delegazione russa il 9 marzo 2002, la rete di al-Qaeda poteva contare sulle sue 55 basi in Afghanistan anche sul supporto di militanti ceceni, nonché europei e cinesi, addestrati da istruttori arabi (Mastronardi \& Leo, 2005).
Nel tentativo di far luce sull'identità del jihadista nei suoi tratti più frequenti sono risultati utili alcuni recenti studi, come il modello "Dria" di Orsini (2016) per quanto riguarda le fasi e le motivazioni che portano alla radicalizzazione, il quale tenta di rispondere ad un comune quesito come quello che pone il problema di cosa possa muovere i terroristi in nome di Allah. Altri studi presi in considerazione sono l'analisi "Pirus" del National Consortium for the Study of Terrorism and Responses to Terrorism (START) dell'Università del Maryland (2018) e, infine, gli studi di Ispi a cura di Vidino, Marone \& Entenmann (2017) e Marone \& Vidino (2018). Il primo studio del think tank milanese analizza i principali tratti riscontrati nei 65 "combattenti di Allah" identificati nei 51 attacchi portati a termine in Europa e Nord America tra il giugno del 2014, quando è stato proclamato il Califfato, e il giugno 2017, mentre il secondo cerca di comprendere l'identità dei 125 foreign fighters legati all'Italia presenti nella lista stilata dal Ministro dell'Interno a ottobre 2017.

Ciò che sicuramente accomuna il jihadista e il terrorista considerato da una prospettiva generale è l'assenza di psicopatologie rilevanti che spingano l'individuo ad agire in nome di un'ideologia, di un gruppo terroristico e ad uccidere. I terroristi ragionano come tutte le altre persone e sono dotati di razionalità e intenzionalità (Orsini, 2016).

Il dataset dell'analisi "Profiles of Individual Radicalization in the United States", o "Pirus", condotta dal consorzio START (2018) dell'Università del Maryland riporta che su 511 profili di jihadisti analizzati ${ }^{2}$ negli Stati Uniti tra il 1948 e il 2018, 435 presentano l'assenza di psicopatologie. Lo studio aggiunge inoltre che soltanto una parte dei restanti 76 individui ha riscontrato malattie mentali professionalmente diagnosticate. Questi dati stanno a significare che almeno per la maggior parte dei terroristi islamisti "americani", la componente psicopatologica non può essere considerata come causa principale o rilevante ai fini della radicalizzazione e dell'azione terroristica.

L'analisi dell'Ispi condotta da Marone \& Vidino (2018), realizzata sui profili individuali dei 125 foreign fighters italiani presenti nella lista stilata dal Ministro dell'Interno a ottobre 2017, conferma come l'assenza di psicopatologie possa essere una caratteristica rintracciabile anche nei "combattenti italiani" che aderiscono alla causa jihadista. L'analisi riporta che soltanto un individuo sul totale del campione risulta aver avuto una storia nota di disturbi psichici al momento della partenza verso le zone di conflitto mediorientali. Gli autori, in ogni caso, affermano come il tema sia complesso e delicato in quanto spesso mancano dati certi sulla salute mentale dei foreign fighters e non si può escludere con certezza la totale assenza di soggetti affetti da psicopatologie.

Tuttavia, l'ultimo report annuale dell'Europol (2020) "Terrorism Situation and Trend Report" riporta come, nel caso in cui si riscontrino terroristi con psicopatologie mentali, risulta arduo e a volte impossibile stabilire se le loro azioni siano da categorizzare come attacchi terroristici o manifestazioni di violenza politica. Alcuni soggetti presentanti dei disturbi mentali, infatti, possono mostrare segni di radicalizzazione che sono una conseguenza della loro psicopatologia, piuttosto che di una convinzione ideologica. Ciononostante, non è da escludere che un individuo affetto da qualche disturbo mentale possa emulare le azioni e il

2 Lo studio analizza i profili 2.226 terroristi suddivisi in base alle ideologie di fondo, ovvero estrema destra, estrema sinistra, motivazioni personali e islamismo. Per i fini di questo elaborato, verrà isolata e presa in considerazione nel database soltanto la popolazione riguardante gli islamisti, per un totale di 511 individui. 
modus operandi di un gruppo terroristico. In questo contesto, la diffusione di parole d'odio, le minacce contro il "nemico" o le fake news sui social media potrebbero condurre gli individui mentalmente instabili o facilmente influenzabili a commettere atti di violenza ideologicamente motivata che possono essere categorizzati come atti terroristici

Orsini (2016), nell'affrontare il problema di una presunta malattia mentale, afferma che un ruolo decisivo viene giocato piuttosto dall'educazione jihadista, rivolta a creare quella che lo studioso chiama "mentalità a codice binario", ovvero un tipo di mentalità educata a ridurre la complessità del reale a due sole categorie, ovvero il Bene e il Male. Quando le televisioni di tutto il mondo hanno riportato la notizia della decapitazione del volontario britannico David Haines nel 2014 nel deserto della Siria, gli occidentali hanno pensato che questo atto così orribile e violento fosse conseguenza di una psicopatologia. Secondo l'esperto, al-Baghdadi non è una mente malata, bensì una "mente educata". Volendo fornire un esempio e analizzando questo specifico caso, mentre la società occidentale distingue tre categorie, ovvero i soldati occidentali, i jihadisti e i volontari occidentali che si recano in Siria per aiutare i musulmani colpiti dalla guerra, l'educazione jihadista nega infatti l'esistenza di una terza categoria oltre a quelle del Bene, i jihadisti, e del Male, gli occidentali. Il caso Haines dimostra come nell'ideologia jihadista il fatto che il volontario britannico sia arrivato in Siria per aiutare musulmani e per fare del bene alla comunità, non lo esuli dall'essere considerato infedele in quanto occidentale e qualunque tentativo di modificare la dicotomia Bene-Male è considerato un oltraggio al Corano.

Questo è possibile in quanto ogni sistema di credenza religiosa crea dei confini simbolici che le consente di marcare la differenza rispetto all'ambiente circostante e distingue ciò che è possibile credere, al suo interno, da ciò che ritiene incredibile, al suo esterno. Il campo delle possibilità del credere può essere ampio o ristretto, tollerante e flessibile o rigido e impenetrabile. In questo contesto, è quindi possibile collocare la dicotomia tra conformità e devianza. Le religioni, in quanto sistemi che si organizzano come un sapere e un potere basati sulla pretesa della verità, devono continuamente presidiare i loro confini simbolici che li distinguono dagli altri sistemi, e, di conseguenza, classificare tutto ciò che ricade al di fuori di esse o ne minacci l'integrità (Pace, 2007).

Inoltre, l'antropologo francese Van Gennep, influenzato dalla scuola di Durkheim, ritiene che ogni società abbia bisogno di organizzarsi delimitando, verso l'esterno, i confini che la distinguono da un'altra, oltre che, verso l'interno, segnando e marcando lo spazio sociale in base a competenze, col fine di garantire ordine nei rapporti sociali e di potere (Pace, 2007).

Orsini (2016), quindi, riporta testualmente le parole riportate dal primo numero di "Dabiq", la rivista ufficiale dello Stato Islamico: "Il mondo è diviso in due campi e due trincee, il campo dell'Islam e della fede e il campo dei miscredenti e degli ipocriti, il campo dei musulmani e dei mujaheddin e il campo degli ebrei, dei crociati, dei loro alleati e di tutte le nazioni e le religioni della miscredenza che sono tutte guidate dagli Stati Uniti e dalla Russia, e che sono mobilitate dagli ebrei" (pag. 131). Quello che emerge è che il jihād islamico è innanzitutto un modo di pensare e di guardare il mondo, ed è un insieme di principi educativi. Sarebbe quindi del tutto fuorviante ai fini di una buona analisi considerare il jihadista come una persona che agisce facendo stragi perché affetto da psicopatologie mentali. L'educazione, che è alla base dell'organizzazione della società, deve raggiungere i giovani attraverso un processo di indottrinamento ma per farlo la società stessa deve essere rigida e chiusa.

Per favorire questo tipo di educazione, ad esempio, al-Qaeda ha creato un'università online per le scienze del jihād, che offre varie specializzazioni quali quelle in "jihād elettronica”, "jihād psicologica", "tecnologia degli esplosivi" e "tecnologia dell'autobomba" (Mastronardi \& Leo, 2005).

Il sociologo Pellicani (2002) quindi individua otto pilastri portanti della "società chiusa", i quali possono essere applicati anche alla società jihadista: sacralizzazione della tradizione, per cui tutti i valori e costumi della società assumono un carattere sacro e ogni deviazione da questi viene considerata un sacrilegio; isolamento, secondo cui la società chiusa deve evitare ogni contatto con le altre società per evitare il "contagio"; autarchia, per cui la società chiusa deve rinunciare alla cose del mondo e bastare a se stessa; ipersocializzazione, ovvero l'individuo che fa parte della società chiusa deve essere spogliato da ogni autonomia di pensiero ed essere conformista; ortodossia, per cui ogni credenza deve essere conforme alla dottrina, ai dogmi e alle istituzioni dominanti; olismo, secondo il quale il tutto deve prevalere sulle singole parti; centralizzazione politica, per cui è importante che il potere confluisca in un unico centro per preservare la tradizione e impedire il mutamento; misoneismo, per cui la società chiusa rifiuta le novità in ogni campo perché l'uomo "creativo" costituisce un pericolo.

La mentalità a codice binario possiede cinque caratteristiche fondamentali, ovvero l'attesa della fine, il catastrofismo radicale, l'ossessione della purezza, l'identificazione del maligno e l'ossessione per la purificazione. Molti purificatori del mondo sono stati individuati prima del crollo dello Stato Islamico nei territori da esso controllati e in Arabia Saudita, Stato promotore del wahabismo. Tuttavia, risulta apparentemente molto più arduo capire il motivo per cui soggetti occidentali, istruiti in modo liberale, decidano di diventare dei purificatori del mondo. La motivazione di ciò può essere ricondotta ad un percorso sociopsicologico che porta il soggetto a distaccarsi dai valori dell'ambiente circostante, creando così una persona "nuova" (Orsini, 2016).

L'adesione ad un gruppo terroristico come l'IS può essere considerata infatti come la risposta ad una domanda di appartenenza non soddisfatta e alla necessità di esprimere delle rivendicazioni che non hanno trovato altra possibilità di manifestazione (Lombardi, 2016).

De Luca (2002) sostiene come alla base della scelta di un soggetto di abbracciare un'ideologia fondamentalista vi sia la soddisfazione di sei bisogni essenziali. Il primo bisogno è di sicurezza, ovvero l'eliminazione dell'incertezza. In questo senso, il Corano, secondo la visione fondamentalista, deve essere seguito alla lettera perché la strada di Maometto viene ritenuta quella giusta da seguire. Il secondo bisogno è quello di autorità, ovvero di sottostare alle imposizioni delle Sacre Scritture o di una personalità carismatica la cui autorevolezza è considerata indiscutibile, come nel caso dei seguaci di Bin Laden. Il terzo bisogno è di identificazione col gruppo di appartenenza e con la sua ideologia. Nel caso del fondamentalismo islamico l'identificazione è totale, tanto che il soggetto è disposto anche a sacrificare la propria vita. Il bisogno di ancoramento rappresenta il quarto bisogno. L'individuo necessita di un sostegno, materiale e psicologico, da parte del gruppo terroristico di appartenenza, come nel caso dell'aiuto economico che viene garantito alle famiglie dei martiri islamisti. Il quinto bisogno è quello di perfezione. Il fondamentalista crede che i propri comportamenti siano perfetti e giusti e che, per tale ragione, egli avrà una ricompensa. L'ultimo bisogno è di semplicità, ovvero basare la propria vita e le proprie azioni su pochi e chiari concetti.

Queste necessità esistono in ogni individuo, ma nel caso di coloro che abbracciano il fondamentalismo islamico, essi hanno una presa maggiore e spingono il soggetto verso idee e comportamenti estremisti e a aderire ad un gruppo terroristico (De Luca, 2002). Picozzi (2016) sottolinea quindi come risulti più interessante e sicuramente più utile concentrarsi sui processi che portano $\mathrm{i}$ 
potenziali jihadisti ad abbracciare la causa terroristica anziché soffermarsi sulla delineazione di un profilo generale.

Orsini (2016) ha elaborato un modello molto importante per il fine del presente lavoro che riassume le tappe che scandiscono il "viaggio" dell'individuo verso la morte jihadista, chiamato "modello Dria", acronimo delle 4 fasi che costituiscono il percorso stesso, ovvero disintegrazione dell'identità sociale, ricostruzione dell'identità sociale, integrazione e alienazione dal mondo circostante. Il modello, utile anche per comprendere come siano possibili il processo della radicalizzazione per soggetti occidentali e la continua alimentazione del fenomeno dei foreign fighters, si fonda sul presupposto che i terroristi non sono dei soggetti con psicopatologie, che le fasi che attraversano sono le stesse che attraversano anche tutte le altre persone che non diventano terroristi e che alQaeda e IS sono prima di tutto fenomeni ideologici, idee, pensieri, rappresentazioni del mondo e schemi cognitivi di attribuzione mentale, i quali servono al soggetto a semplificare la complessità del reale nei suoi collegamenti causa-effetto. Quindi, alla luce di ciò, è possibile affermare che terroristi si diventa e non si nasce.

Prima di trattare ogni singola fase del modello, risulta utile premettere l'esistenza di una distinzione tra marginalità sociale ed emarginazione sociale. La prima crea l'individuo marginale, la seconda l'emarginato sociale. Mentre l'emarginazione sociale può essere ricondotta ad una condizione di privazione oggettiva, come ad esempio ad individui poveri e con scarse aspettative di vita, la marginalità sociale è una condizione sociopsicologica che può colpire anche individui medio o alto borghesi, di status elevato e con grande disponibilità economica. Quest'ultimo processo porta ad una particolare condizione esistenziale e genera una forte tensione emotiva che fa sì che anche un individuo di alto rango possa sentirsi estraneo rispetto alla società in cui vive. Questa condizione di disagio, generalmente orientata nei ragazzi musulmani occidentali di seconda e terza generazione da valori religiosi in contrasto con la mentalità capitalista, può colpire qualsiasi soggetto e non solo coloro che poi sceglieranno di diventare jihadisti. Tutto ciò comporta un distaccamento dell'individuo dai valori dominanti della società rendendosi disponibile ad abbracciarne di nuovi. È fondamentale quindi non confondere o sovrapporre i due concetti così come risulta erroneo pensare che la marginalità sociale sia in ogni caso sinonimo di terrorismo. Infatti, è importante evidenziare che il poter affermare che i jihadisti "cresciuti in casa" siano ragazzi disorientati non significa affermare che tutti i ragazzi disorientati poi diventeranno terroristi (Orsini, 2016).

Salvatori (2018) aggiunge come questo processo comporti l'alimentazione del fenomeno dei lupi solitari, ragazzi giovani segnati dallo sradicamento culturale, penalizzati da meccanismi di selezione e integrazione oppure semplicemente vittime di alienazione psicologica.

De Pasquali (2016), a questo proposito, afferma come inevitabilmente il confronto tra due culture diverse generi conflitto e, ad oggi, l'immagine di una società multiculturale e di una piena integrazione resti ancora un miraggio.

La condizione economica, pertanto, non rappresenta una variabile determinante nella scelta di diventare un terrorista. Qualsiasi individuo può cadere in una condizione riconducibile alla marginalità sociale a prescindere dalla sua disponibilità di denaro. Volendo riportare un esempio, i fratelli Kouachi, due degli autori della strage nella redazione di Charlie Hebdo di Parigi del 7 gennaio 2015, erano ragazzi poveri con un difficile passato e un'infanzia dolorosa. Dall'altro lato, Abdelhamid Abaaoud, la mente degli attentati di Parigi del 13 novembre 2015, non viveva in una condizione di povertà, in quanto frequentò una prestigiosa scuola cattolica a Bruxelles e beneficiò di privilegi non accessibili per molti suoi coetanei (Orsini, 2016).
Il nesso tra condizione economica e processi di radicalizzazione è un tema da molto tempo oggetto di discussione e dibattito da parte degli esperti. Nello studio di Marone \& Vidino (2018) relativo ai foreign fighters "italiani", emerge che, sebbene non siano disponibili informazioni certe e dettagliate sui soggetti prima della partenza verso le zone di conflitto, il benessere economico non risulta rappresentare una discriminante fondamentale nella decisione di radicalizzarsi. Nello studio sono analizzati infatti profili di 125 foreign fighters con una condizione economica tanto agiata quanto con capacità economiche non particolarmente elevate. Informazioni indirette sulla condizione economica dei soggetti possono essere fornite dal tipo di lavoro svolto prima della partenza e dal livello di istruzione. La maggior parte dei foreign fighters considerati risultava disoccupata, per il $34,4 \%$ del totale, o impiegata in lavori manuali, per il 44,8\%. La minoranza svolgeva lavori d'ufficio, pari all' $8 \%$.

Sebbene si sia spesso portati a pensare che la motivazione principale dell'adesione ad un gruppo terroristico sia la povertà, osservazione dovuta dalla reale provenienza di molti foreign fighters da condizioni di precarietà economica o di assoluta povertà, è altresì evidente e incontestabile che non sono pochi coloro che invece appartengono a ceti-medio alti e provengono da paesi industrializzati (Teti, 2018).

Questa tesi viene rafforzata dai dati dell'analisi condotta dal consorzio START (2018), la quale riporta come la maggior parte dei 511 jihadisti profilati negli Stati Uniti appartengano ad uno strato sociale medio, sia per quanto riguarda la fase di vita relativa all'infanzia che a quella adulta. In numero minore sono presenti, tuttavia, anche soggetti di classe sociale bassa e alta. Molti di essi hanno raggiunto un livello d'istruzione medio-alto.

Diversi studi sulla componente economica e le variabili direttamente collegate ad essa, come l'istruzione, quindi, presentano dati altamente eterogenei e a volte divergenti.

Teti (2018) riporta le conclusioni delle ricerche di studiosi come Paul Wilkinson della St. Andrews University, secondo cui la povertà e la privazione materiale ed economica subite dall'individuo non si traducono immediatamente in aggressività e violenza. Ad esempio, la popolazione povera del Terzo Mondo non sarebbe psicologicamente preparata a lotte rivoluzionarie o alla partecipazione politica, a causa della quotidiana lotta alla sopravvivenza.

Zoja (2018) afferma come sia inevitabile considerare una serie di componenti economiche tra le cause della radicalizzazione, ma nello stesso tempo sia insufficiente in quanto altrimenti ci sarebbe stato un infinito numero di radicalizzazioni terroristiche, in infiniti luoghi e infinite generazioni. Si tratta piuttosto di un problema di scarsa appartenenza alla comunità da parte della società occidentale, in quanto sbilanciata troppo verso i valori e gli interessi personali.

Orsini (2019) riporta la teoria di Marc Sageman, secondo cui il fatto che i terroristi siano uomini poveri e disperati, provenienti da Paesi del Terzo Mondo, vulnerabili al lavaggio del cervello, sia soltanto uno stereotipo molto diffuso. Se così fosse, infatti, il Terzo Mondo dovrebbe essere colmo di terroristi ma, afferma Sageman, così non è. I terroristi sono giovani di classe media con buon livello d'istruzione, cresciuti in famiglie religiose con valori forti e positivi, preoccupati per il bene delle loro comunità.

Da queste considerazioni è quindi possibile affermare come la condizione economica e le variabili ad essa collegate, come il livello d'istruzione e l'impiego lavorativo, non siano determinanti al fine della marginalità sociale e della radicalizzazione.

Teti (2018) aggiunge come piuttosto la disuguaglianza sociale e l'umiliazione siano le motivazioni principali citate dai terroristi per fornire una spiegazione alla loro scelta di vita. Molti sono stati infatti i combattenti che ritengono di aver subito nel corso della loro vita umiliazioni o soprusi dalla società o dalle istituzioni. 
Il primo passo del processo verso la marginalità sociale e verso l'adesione alla causa terroristica è contraddistinto infatti da un trauma subito o da una condizione di sofferenza socio-psicologica latente nel soggetto, la quale esplode improvvisamente a causa in un particolare evento negativo, distruggendo lo stato di equilibrio psicologico e portando così la vittima verso una forte tristezza o nel tunnel della depressione. Alcuni degli eventi scatenanti possono essere il tradimento coniugale, il licenziamento dal lavoro, la detenzione in carcere, un ingiusto processo, l'essere parte di una famiglia violenta, essere oggetto di discriminazioni e, più in generale, la frantumazione del sogno a cui si è dedicata l'intera vita. Quando uno di questi eventi si abbatte sulla psicologia della persona, questa può cadere nella depressione e diventare dipendente dai farmaci oppure iniziare a riflettere sulla sua condizione e a ritenere di vivere in una società sbagliata, dove l'individuo stesso non si riconosce più, abbracciando così una nuova ideologia (Orsini, 2016).

L'individuo marginale può quindi essere definito come "una persona che è nel mondo senza essere nel mondo, per una scelta alimentata da un'ideologia che si basa sull'idea che la società è un luogo contaminato da cui bisogna separarsi" (Orsini, 2016, pag. 152).

Una volta presa la decisione di intraprendere questo percorso, si entra nella prima fase del "modello Dria" di Orsini, ovvero la disintegrazione dell'identità sociale, un processo psicosociologico che consiste nell'annientamento della propria identità per adottarne una nuova. Una delle caratteristiche tipiche degli individui che entrano in questa fase è che l'impegno di questi soggetti a voler uscire dalla loro condizione esistenziale si traduce spesso nella disponibilità ad aprire i propri orizzonti a nuove visioni del mondo, che nella maggior parte dei casi si traduce a sua volta in una conversione spirituale, religiosa o ideologica. Secondo l'autore, quindi, i soggetti che "disintegrano" la loro identità sociale, lo fanno essendo "imprenditori di sé stessi" e, dunque, non necessariamente a causa di un'operazione di brainwashing da parte di un soggetto terzo (Orsini, 2016).

La seconda fase del modello di Orsini (2016) prevede la ricostruzione dell'identità sociale. In questa fase, l'individuo, in cerca di una via d'uscita alla sua condizione di inadeguatezza nella società, cerca un punto di riferimento, che può essere individuato sia in una persona che in una ideologia. Con questa fase avviene quindi una conversione esistenziale. La maggioranza dei ragazzi occidentali diventati jihadisti, continua l'autore, si è convertita dal Cattolicesimo, o Cristianesimo in generale, all'Islam. Da qui nascono i "terroristi occidentali", la cui conversione, laddove spontanea e non imposta, è dovuta ad un originario sistema di credenze non più riconosciuto, che li porta all'adozione di una nuova identità, di un nuovo nome, come testimonianza di una "rinascita".

Nella sociologia delle religioni, la religione è infatti considerata come il tentativo più ambizioso di mettere ordine nella società, poiché pretende di definirne tutte le sfumature dell'esistenza umana e i legami sociali a partire da un principio unitario sovraordinato (Pace, 2017).

È tuttavia importante sottolineare che non tutti coloro che entrano in una fase di disintegrazione dell'identità sociale poi abbracciano l'ideologia jihadista. Dalla condizione di smarrimento esistenziale si può infatti uscire in diversi modi, come ad esempio attraverso l'innamoramento o l'identificazione in un collettivo, come in una squadra sportiva. Importanti sono fattori come l'amore, la comprensione e la solidarietà. Tuttavia, coloro che decidono di abbracciare un'ideologia radicale, ovvero un sistema di pensiero che pretende di regolare tutti gli aspetti della vita quotidiana in maniera rigida e intollerante, adottano una dottrina estremamente rigida che orienta inevitabilmente la persona, non permettendo così alla stessa di "smarrirsi" o disorientarsi nuovamente. L'ideologia islamista può essere considerata in questo senso un'ideologia iperradicale, in quanto non rappresenta soltanto un modo di vedere la politica e la storia, ma costituisce un insieme di precetti che regola la vita quotidiana in tutti i suoi dettagli e che distingue soltanto due sfere, il Bene e il Male. L'ideologia jihadista, dello Stato Islamico o di al-Qaeda, rappresenta una sorta di "trappola", in quanto da un lato attribuisce nuovamente valore alla vita di coloro che l'avevano perso e dall'altro attribuisce al contrario un valore molto basso alla vita altrui (Orsini, 2016).

La decisione di salvare sé stessi attraverso l'adozione di una ideologia come quella jihadista può essere considerata in ottica spinoziana come una buona azione, in quanto per un essere umano la buona azione si traduce con tutto ciò che è utile per l'autoconservazione (Pace, 2007).

La terza fase del modello "Dria" è la fase dell'integrazione in una setta rivoluzionaria, come una cellula terroristica, una setta satanica ma anche una comunità religiosa fondata sul rifiuto della violenza, in quanto l'identità sociale può essere ricostruita anche attraverso ideologie o concezioni del mondo le cui caratteristiche portanti sono l'amore e la tolleranza (Orsini, 2016).

Nel XIX secolo il positivista Comte riteneva che ogni società, così come un organismo, ha bisogno di trovare un punto di equilibrio attorno a valori condivisi e visioni del mondo comuni. La religione per Comte soddisfa i bisogni profondi dell'uomo di carattere cognitivo e comportamentale e contribuisce così alla coesione e funzionamento della società. Successivamente la religione, secondo il funzionalismo classico di Durkheim, e il neo-funzionalismo di Parsons e Luhmann, svolge un ruolo unitario, cioè quello di costituire un potente fattore di stabilizzazione sociale, poiché riesce fornire, a parte della società o alla sua totalità, profondi meccanismi di riduzione della contingenza psicologica, sociale e politica. Clifford Geertz, uno dei maggiori antropologi contemporanei, supera questo approccio sistemico assumendo, per di più, che la religione non rispecchia tanto l'ordine sociale ma lo crea, e la definisce come un sistema di simboli che costruisce un coerente ordine di credenze capace di orientare le azioni degli esseri umani (Pace, 2007).

Secondo ancora i teorici della "rational choice theory, ogni essere umano compie delle scelte basate sul calcolo dei costi e benefici, col fine di raggiungere il massimo dei vantaggi possibili. La teoria della scelta razionale propone quindi di considerare la religione non tanto in prima istanza come un modo per legare la società, bensì soprattutto come frutto di una scelta razionale, distaccandosi così dal funzionalismo. Ciò significa che tale scelta è razionale nel senso che risponde alle esigenze proprie di un individuo e al suo modo di valutare costi e benefici (Pace, 2007).

In questa fase del modello, l'individuo va alla ricerca di coloro che hanno le sue stesse idee. Nel caso delle ideologie radicali come quella jihadista, il fatto di aver scoperto la verità ultima sul significato della storia e della convivenza umana, porta il soggetto alla ricerca di un gruppo in cui si possa rispecchiare ed è proprio in questa tappa del processo che vi sono i primi contatti con organizzazioni fondamentaliste. Il contatto può avvenire fisicamente o virtualmente, ovvero tramite il web. Tuttavia, è possibile entrare in un gruppo anche solo attraverso la fantasia e quando ciò accade si entra a far parte di una "comunità immaginata" e l'individuo che ne accede pretende di essere trattato come un membro del gruppo. È questo il caso di quei simpatizzanti che entrano in contatto con gruppi fondamentalisti come l'IS attraverso internet e subito iniziano a fregiarsi di simboli della comunità senza mai partire realmente per le zone di conflitto. Di conseguenza, è possibile entrare a far parte di un gruppo e assumerne i modelli di comportamento anche senza mai incontrare fisicamente i suoi membri. In ogni caso, sia che si tratti di un'unione "fisica" o "immaginata" ad un gruppo, l'adesione deve essere considerata reale, laddove per reale si intende tutto ciò che è esistente per 
l'individuo in base al suo punto di vista e, conseguentemente, influenza le sue azioni (Orsini, 2016).

Una volta ottenuto l'accesso alla comunità jihadista, il "nuovo combattente di Allah" comincia ad acquisire in toto gli usi e costumi dell'organizzazione. È fondamentale sotto questo punto di vista l'allontanamento dagli occidentali, ritenuti dagli aspiranti jihadisti come ripugnanti, attraverso un processo di esclusione ed autoesclusione. Si entra in questo modo nella quarta fase del modello "Dria", che prevede l'alienazione dal mondo circostante. In quest'ultima parte del processo verso la morte jihadista avviene quella che l'autore chiama "la fine del feedback negativo", il quale si annulla con l'ingresso in una cellula jihadista. Per chiarire questa espressione, viene utilizzato l'esempio di Mohammed Bouyeri, terrorista marocchino-olandese condannato all'ergastolo, che il 2 novembre 2004 decide di uccidere il regista Theo Van Gogh nel centro di Amsterdam, colpevole di aver realizzato un documentario che criticava la cultura islamica. La volontà di Bouyeri di assassinare il regista, oramai entrato a far parte del gruppo terroristico olandese "The Hofstad Network", non ha visto obiezioni da parte degli altri membri, in quanto assorbiti irreversibilmente nel mondo e nell'ideologia jihadista e, di conseguenza, soggetti ad una convergenza di opinioni. Laddove ogni membro di un gruppo assecondi gli intenti criminali di un altro, si è entrati a far parte di un'organizzazione in cui c'è totale assenza di feedback negativo basato sui valori morali del mondo esterno, inteso come di un'opposizione all'intento criminale che normalmente nella società domina la coscienza collettiva (Orsini, 2016).

Il modello "Dria" di Orsini dimostra come terroristi si diventi e mette in luce tutti quei meccanismi sociali e psicologici di avvicinamento al mondo jihadista a cui sono soggetti tutti quegli individui che precipitano in una condizione disagevole. Non c'è spazio quindi per un effetto determinante da parte della componente psicopatologica, seppur a volte individuabile negli individui analizzati, come dimostrato dagli studi sopra riportati.

Inoltre, appare utile focalizzare l'attenzione anche su altre variabili individuabili dalle analisi sui profili dei terroristi islamisti come l'età, il sesso, la cittadinanza e il paese di nascita, lo status di immigrazione, $\mathrm{i}$ trascorsi criminali e i legami operativi con i gruppi terroristici.

Per quanto riguarda l'età dei jihadisti, dagli studi di Vidino, Marone \& Entenmann (2017) emerge come la popolazione jihadista risulti giovane al momento del reclutamento. L'età media dei 65 attentatori, autori dei 51 attacchi portati a termine in Europa e Nord America tra il giugno 2014 e il giugno 2017, è pari a 27,3 e, in generale, si assiste ad una tendenza verso la radicalizzazione di ragazzi sempre più giovani. Il coinvolgimento di soggetti minorenni negli attacchi terroristici risulta molto contenuto con soltanto cinque casi, mentre circa un terzo del campione considerato risulta avere più di 30 anni, elemento sorprendente visto il trend attuale delle reclute del terrorismo.

Relativamente invece ai 125 foreign fighters italiani analizzati da Marone e Vidino (2018), l'età media al momento della partenza è di 30 anni, mentre la fascia di età principale è quella tra 25 e 33 anni. Il foreign fighters più giovane rilevato è risultata essere una ragazza di 16 anni, figlia di una convertita italiana e appartenente ad un nucleo familiare di origini italo-marocchine partito dal Belgio. La minorenne, con cittadinanza italiana, si è sposata in Siria con un combattente algerino, emiro dello Stato Islamico e, in seguito, ha fatto ritorno in Belgio e viene arrestata all'aeroporto di Bruxelles insieme alla madre perché sospettate di voler partire nuovamente per la Siria.

Sulla base degli studi, tuttavia, si stima come il numero di bambini partiti assieme ai genitori o conoscenti possa essere superiore a sette. Al contrario, il combattente più anziano è risultato essere un uomo marocchino di 52 anni partito dal Regno Unito. L'uomo aveva vissuto a Roma per circa vent'anni ed era iscritto al "Movimento della Gioventù Islamica" marocchino. In media, i dati relativi all'età dei foreign fighters italiani appaiono leggermente superiori a quelli degli altri paesi europei, dove la maggior parte dei combattenti è contenuta nella fascia d'età tra i 18 e 30 anni.

Il dataset dell'analisi "Pirus" realizzato dal consorzio START (2018) dell'Università del Maryland riporta i dati relativi all'età dei 511 jihadisti americani considerati al momento dell'attacco o dell'arresto. La metà dei combattenti, pari a 256 soggetti, è contenuta nella fascia d'età tra i 20 e 30 anni, mentre soltanto 58 sono i ragazzi più giovani o minorenni. Le altre fasce di età più rappresentate sono quelle tra i 30 e 40 anni con 122 soggetti e quella tra i 40 e 50 anni con 48 . Soltanto 20 individui sono essere risultati avere più di 50 anni, la maggior parte collocabili della fascia d'età tra i 50 e 60 anni.

Gli studi sopra menzionati convergono quindi nelle loro analisi e conclusioni circa l'età dei combattenti, asserendo che per la maggior parte dei casi si tratta di soggetti giovani o relativamente giovani. La fascia d'età più rappresentata è quella che va dai 20 ai 30 anni, con delle eccezioni più o meno corpose numericamente nelle fasce d'età più avanzate.

Relativamente a ciò, Zoja (2018) ritiene come la giovane età renda i soggetti più psicologicamente vulnerabili ed esposti alle seduzioni del radicalismo e alla propaganda.

Per quanto riguarda la variabile relativa al sesso del jihadista, dalle ricerche emerge all'unanimità come $\mathrm{i}$ jihadisti di sesso maschile siano in netta maggioranza rispetto alle donne, seppur quest'ultime ricoprano ruoli non secondari all'interno delle organizzazioni terroristiche e il loro tasso di adesione sia in progressivo aumento. Risulta errato, quindi, pensare il mondo jihadista come un mondo esclusivamente al maschile. Tradizionalmente, il ruolo delle donne nel terrorismo di matrice jihadista è rimasto essenzialmente confinato ad attività ausiliarie e di supporto, lontane dal combattimento. I compiti svolti per la maggiore dalle donne sono relativi al reclutamento, alla logistica e alla promozione di attività terroristiche (Alexander, 2016). Nell'ottica della propaganda sono coinvolte giovani donne occidentali che, attraverso i social, raccontano la loro vita di donne emigrate nel Califfato e condividono i loro diari da migranti. Se il reclutamento di jihadiste ha visto un'impennata dopo la nascita ufficiale del Califfato, il fenomeno vero e proprio ha origini ben più antiche. Già da prima dell'inizio delle guerre siriane le donne accompagnavano i jihadisti in Afghanistan e in Siria, tanto che già in quel periodo si cominciò a parlare di "sex jihād", con riferimento ai flussi di donne (Lombardi, 2016).

Lo Stato Islamico ha da tempo progettato in questo senso una strategia che prevede il reclutamento tra le sue fila di un ingente numero di jihadiste da impiegare nelle attività di reclutamento online e offline, di costruzione dell'apparato statale, come ad esempio insegnanti o infermiere, e di sorveglianza di altre donne destinate alla schiavitù tramite la Brigata al-Khanssaa, unità esclusivamente femminile che rappresenta una sorta di polizia morale con sede a Raqqa. Nei casi di partecipazione ad attività terroristiche con ruoli più attivi, invece, le donne sono impiegate in combattimento da gruppi jihadisti come al-Shabaab e Boko Haram fino anche alla realizzazione di attacchi suicidi (Vidino, Marone \& Entenmann, 2017).

Risulta ora interessante ai fini della delineazione dell'identità jihadista approfondire le variabili riguardanti il paese di nascita, la cittadinanza, lo status legato all'immigrazione e i legami con organizzazioni terroristiche, variabili che consentono di comprendere non solo le origini dei terroristi, ma anche la natura degli attacchi realizzati in Europa negli ultimi anni.

Ai fini di questo lavoro è necessario soffermarsi sul nesso tra 
terrorismo jihadista e immigrazione, connessione che viene spesso esaltata dai media contribuendo ad alimentare l'idea di uno straniero pericoloso e terrorista. Il terrorismo islamista viene alimentato da continui e incontrollati flussi di immigrati, provenienti soprattutto dall'area del Mediterraneo e dai Balcani. L'incremento di queste ondate migratorie ha infatti accompagnato in passato l'intensificarsi degli attacchi terroristici non solo nelle zone di conflitto, come in Siria ed Iraq, ma anche in Europa. È possibile affermare che esiste infatti un importante sodalizio tra immigrazione clandestina ed estremismo islamico, poiché appare altamente probabile l'infiltrazione di miliziani jihadisti tra gli immigrati che arrivano in Europa. Nonostante le prove di tentativi di infiltrazione attraverso i flussi di migranti, alcuni studiosi ritengono al contrario improbabile l'utilizzo delle rotte migratorie da parte delle organizzazioni jihadiste come strumento di infiltrazione dei terroristi all'interno del territorio italiano ed europeo, a causa del rischio che l'attraversamento via mare creerebbe per il capitale umano jihadista, ritenuto per le organizzazioni estremamente prezioso. Ciò che ora è fondamentale evidenziare è che, secondo gli studiosi, il vero pericolo non proviene in realtà dagli sbarchi di immigrati, bensì dai foreign fighters e dai terroristi "home made", ovvero soggetti stranieri, solitamente di seconda o terza generazione, che hanno ottenuto la cittadinanza europea e che proprio in Europa si nascondono, operando indisturbati (De Pasquali, 2016).

I militanti di seconda e terza generazione, a differenza dei terroristi di prima generazione che giungono alla violenza in modo graduale in quanto hanno bisogno di tempo per rompere i legami con i valori acquisiti in famiglia, crescono in un ambiente che è già stato radicalizzato dai militanti di prima generazione e vengono socializzati alla violenza già da bambini e da adolescenti. Questo fattore generazionale è molto importante e utile per comprendere il perché il reclutamento di jihadisti veda sempre di più coinvolti soggetti molto giovani, senza freni inibitori verso le azioni più estreme (Orsini, 2019).

In Italia, gli immigrati di prima generazione sono i soggetti più coinvolti in attività radicali insieme ad un numero crescente di jihadisti autoctoni, cresciuti nel Belpaese, composto da immigrati di seconda generazione, oltre che da convertiti di origine italiana. È importante sottolineare come il numero di soggetti radicalizzati nati o cresciuti in Italia sia molto esiguo e in controtendenza rispetto ad altri paesi europei. La causa di ciò è da ricondurre alla maggiore integrazione di questi stranieri nelle città italiane, evitando così la creazione di "ghetti" nelle periferie, e alla storia migratoria italiana legata ai migranti musulmani. I flussi migratori da paesi a maggioranza musulmana sono iniziati alla fine degli anni Ottanta e all'inizio degli anni Novanta, ovvero molto più tardi rispetto ad altri paesi dell'Europa occidentale (Marone \& Vidino, 2018).

L'Italia è sembrata per ora evitare quindi la pericolosa e rischiosa costituzione di città caratterizzate da periferie dominate dall'emarginazione sociale, fenomeno invece presente in alcune grandi città europee. Per i giovani delle banlieue di Parigi e dei sobborghi londinesi tipicamente popolati da musulmani, e per questo denominati "Londonstan", infatti, l'emarginazione diventa tale da vedere il jihād come l'unica occasione per emergere e uscire dalla condizione di totale anonimato, aggredendo una civiltà della quale paradossalmente aveva precedentemente assorbito i disvalori (Salvatori, 2018). L'Europa non è riuscita ad integrare le seconde e terze generazioni d'immigrati, socialmente ed economicamente, lasciando molti giovani disorientati e incapaci di scegliere tra $\mathrm{i}$ valori secolari dell'Occidente e il patrimonio religioso dei loro paesi originari. Questo conflitto interiore rende particolarmente vulnerabili all'estremismo (Silber, 2012).

La pressione migratoria degli ultimi anni, soprattutto per quanto riguarda l'Italia, proviene da zone ad alta instabilità politica $\mathrm{e}$ sociale e in cui si sono costituite milizie armate legate a gruppi fondamentalisti islamici. La Primavera araba e i conflitti scoppiati in Nord Africa e Medio Oriente hanno provocato il crollo di molti stati, caduti inevitabilmente in uno stato di crisi, e facilitato di conseguenza l'azione dei trafficanti di esseri umani, in quanto gli spostamenti degli immigrati avvengono senza alcun controllo dalla zona sub-sahariana o mediorientale fino le coste del Mediterraneo. Un ruolo chiave riveste per l'Italia il futuro della Libia, paese senza attualmente una stabilità politica e da cui partono barconi di clandestini verso Lampedusa, la maggior parte dei quali provenienti dalla Siria, territorio che era in mano allo Stato Islamico. Già nel 2015, prima del crollo del Califfato, è stata rilevata l'importanza della Libia per al-Baghdadi, utilizzata come punto nevralgico per minacciare 1'Europa (De Pasquali, 2016).

Negli Stati Uniti i dati dell'analisi "Pirus" del consorzio START (2018) mostrano come anche oltreoceano la maggior parte dei jihadisti siano della stessa nazionalità del paese in cui operano, confermando così il trend europeo dei terroristi "home grown".

Venendo ora ai legami con le organizzazioni terroristiche dei jihadisti autori dei recenti attentati in Europa, sul versante operativo è possibile suddividere gli attentati in tre macrocategorie. La prima categoria racchiude tutti gli attacchi perpetrati da individui che hanno ricevuto ordini direttamente dai vertici dello Stato Islamico, corrispondenti all' $8 \%$ di tutti gli attentati. La seconda categoria si riferisce a tutti quegli attentati perpetrati da jihadisti senza alcun legame con gruppi organizzati ma ispirati dal loro messaggio, per una percentuale che si attesta al $26 \%$. La terza categoria infine è riconducibile a tutti gli attentati commessi da soggetti che hanno agito autonomamente ma aventi una qualche forma di connessione con organizzazioni. A quest'ultima categoria sono riconducibili la maggior parte degli attacchi, per una percentuale pari al 66\% (Vidino, Marone \& Entenmann, 2017).

Osservando e interpretando i dati sopra riportati relativi alla condizione legata all'immigrazione e ai legami operativi con lo Stato Islamico e le altre organizzazioni jihadiste, è possibile affermare come la maggior parte degli attacchi terroristici commessi negli ultimi anni in Europa non siano direttamente imputabili allo Stato Islamico e dai suoi vertici, quanto piuttosto a immigrati radicalizzati di seconda e terza generazione, alcuni con un passato da foreign fighters, legati all'ideologia jihadista e in qualche modo a gruppi terroristici ma che hanno agito autonomamente.

Questa lettura conferma gli scritti degli analisti di intelligence, secondo cui, relativamente al Califfato, non esiste alcuna organizzazione internazionale che gestisca i militanti jihadisti col fine di creare un califfato globale e conquistare l'Occidente. In Europa la minaccia jihadista proviene essenzialmente da lupi solitari, ossia da individui che agiscono autonomamente. La rilevanza dell'IS va infatti considerata nell'ambito della regione in cui ha operato sino alla sua caduta. Il vero fine di al-Baghdadi è quello di ricostituire il triangolo sunnita, sfaldatosi tra Siria, Iraq e regioni limitrofe a causa di accordi geopolitici occidentali, ricreando così il Califfato in Medio Oriente (Salvatori, 2018).

L'ultima relazione del Dipartimento delle Informazioni per la Sicurezza (2020) conferma come le azioni di stampo jihadista realizzate in Europa nel 2019, in lieve ripresa rispetto ai precedenti 12 mesi, siano minacce prevalentemente endogene e che hanno visto, in linea di continuità con gli ultimi anni, l'attivazione di lupi solitari. Per tutte, Daesh ha continuato a rappresentare il principale ispiratore, attraverso gli appelli al jihād reiterati dalle "case madri" mediatiche o lanciati e ripostati dai sostenitori sparsi nel mondo, il cui ruolo è parso tanto più rilevante quanto più è andato ridimensionandosi l'apparato propagandistico ufficiale.

Gli attentati realizzati in Europa non vanno quindi letti secondo l'ottica del terrorismo internazionale così come gli attacchi 
dell'11 settembre per mano qaedista, bensì secondo gli schemi del terrorismo domestico indotto di imitazione da parte di terroristi "home made".

Per quanto riguarda invece gli Stati Uniti, il 27\% dei 511 jihadisti non è risultato essere membro di alcuna organizzazione terroristica formale e il $36 \%$ è stato rilevato avere un collegamento con un gruppo informale di estremisti. Il restante 37\% comprende invece terroristi membri di un gruppo organizzato ufficiale e di un movimento politico o gruppo di attivisti (START, 2018).

Osservando i dati appena riportati riguardanti il fenomeno jihadista americano emerge quindi un sostanziale equilibrio nei numeri tra le diverse categorie, evidenziando di conseguenza come anche negli Stati Uniti sia presente un fenomeno di jihadismo autonomo o, comunque, di attentatori con un collegamento a gruppi non facenti capo a organizzazioni terroristiche ufficiali come lo Stato Islamico o al-Qaeda.

Relativamente alla situazione familiare e ai 125 foreign fighters in connessione con l'Italia, circa il $61 \%$ dei soggetti considerati è risultato non essere coniugato al momento della partenza verso le zone di conflitto. Questo dato appare interessante, poiché sembra essere rilevante in questo contesto ciò che gli scienziati sociali chiamano "disponibilità biografica" per fenomeni come il terrorismo e l'attivismo, ovvero l'assenza di vincoli personali che possono aumentare i costi e i rischi della partecipazione a tempo pieno a movimenti o gruppi, come il matrimonio o le responsabilità familiari. In altri termini, i soggetti sposati con figli tendono in genere ad essere meno disposti alla partenza, in quanto da un lato le responsabilità familiari riducono l'ammontare di tempo ed energie a disposizione e, dall'altro, aumentano il peso dei rischi associati a tali azioni. Non sorprende quindi che il fenomeno dei foreign fighters veda coinvolti soprattutto individui giovani celibi senza prole. A livello europeo, le statistiche mostrano dati leggermente diversi rispetto all'Italia a causa di una proporzione maggiore di individui coniugati, almeno per quanto concerne alcuni paesi. Ad esempio, in Germania, su 688 foreign fighters su cui sono disponibili informazioni, il $44 \%$ è costituito da single, il $28 \%$ da soggetti legalmente sposati e il restante $22 \%$ da coniugati attraverso rito islamico. ${ }^{3}$ Nei Paesi Bassi la tendenza che vede un aumento di soggetti sposati viene confermata, con circa il $75 \%$ di foreign fighters su un campione di 78 soggetti risultati coniugati con rito islamico ${ }^{4}$ (Marone \& Vidino, 2018).

La tendenza a non essere coniugati può essere estesa anche agli attentatori in Europa degli ultimi anni con, tuttavia, alcune eccezioni, come ad esempio Amedy Coulibaly, responsabile dell'attacco al supermercato Kosher, e i fratelli Kouachi, ovvero gli attentatori del giornale satirico francese Charlie Hebdo. Amedy Coulibaly è risultato essere legato ad una donna, Hayat Boumedienne, entrambi caduti nella condizione di marginalità sociale che li ha portati in seguito all'adesione all'Islam radicale e all'acquisizione dell'ossessione per la purezza che contraddistingue l'universo mentale jihadista. Anche gli attentatori al giornale francese, ovvero Said e Cherif Kouachi sono risultati essere sposati prima di abbandonarsi totalmente al fondamentalismo islamico e al mondo del terrorismo (Orsini, 2016).

3 Per ulteriori approfondimenti consultare: Heinke D.H., (2017), German Foreign Fighters in Syria and Iraq: The updated Data and Its Implications, CTC Sentinel, Vol.10, n.3, marzo.

4 Per ulteriori approfondimenti consultare: Bergema R., Van Sam M. (2018), Waves of the black banner: An Exploratory Study on the Dutch Jihadist Foreign Fighters in Syria and Iraq, Studies in Conflict \& Terrorism, November.
Come evidenziato precedentemente, la condizione di marginalità sociale può essere causata da un trauma pregresso, altro fattore da tenere in considerazione nella decisione di aderire alla causa jihadista. Negli Stati Uniti il fenomeno assume una dimensione non definibile, poiché per 306 individui su 511 non sono disponibili informazioni riguardanti le esperienze traumatiche dei jihadisti "made in USA" (START, 2018). Tuttavia, maggiori informazioni sono disponibili per gli attentatori in Europa dove il 57\% dei terroristi è risultato avere traumi relativi a trascorsi criminali, mentre $1^{\prime} 82 \%$ era già noto alle autorità prima dell' attacco (Vidino, Marone \& Entenmann, 2017).

Volendo riprendere gli esempi appena citati degli attentatori di Parigi, Amedy Coulibaly nasce in un sobborgo di Parigi, in un complesso diventato presto un luogo di spaccio di droga, traffico di armi, criminalità giovanile, attacchi alla polizia e povertà diffusa. Viene arrestato più volte e condannato per rapina e per i suoi legami col mondo jihadista e, sin dall'infanzia, vive in una famiglia problematica. Anche i fratelli Kouachi vivono un'infanzia tormentata, a causa della morte dei genitori che lasciano Cherif e Said senza soldi e a doversi crescere autonomamente. I due fratelli vengono ospitati in orfanotrofio e crescono a contatto con la piccola criminalità e, come Coulibaly, vengono arrestati più volte per piccoli crimini. Storie di vita tormentate simili a quelle di Amedy Coulibaly e dei fratelli Kouachi compaiono frequentemente nei profili degli attentatori jihadisti e tra le esperienze di questi soggetti sono presenti spesso anche trascorsi criminali e detenzioni in carcere. Le esperienze traumatiche costringono così gli individui a ricercare un nuovo motivo per essere al mondo, spesso individuato nell'ideologia fondamentalista (Orsini, 2016).

È indicativo riportare anche la storia di Mohamed Bouhlel, autore della strage di Nizza il 14 luglio 2016, giorno in cui i francesi celebrano la presa della Bastiglia. Bouhlel è stato un uomo tormentato con una vita colma di fallimenti, come il divorzio, probabilmente per violenze domestiche, e la perdita del lavoro. Il padre è stato testimone dei suoi problemi di alcol, di droga e di depressione (Orsini, 2018). Beaumont (2016), in un articolo per il quotidiano The Guardian, scrive come Bouhlel tra il 2002 e il 2004 è vittima di un violento esaurimento che lo ha portato ad urlare senza motivo e a spaccare gli oggetti improvvisamente. Anche il fratello dell'attentatore ha fornito importanti dettagli sull'attentatore, descrivendolo come un uomo infelice e tormentato. Secondo i familiari, relativamente alla sua vita religiosa, Bouhlel non aveva mai letto il Corano, non era stato mai visto in una moschea e non aveva mai rispettato gli obblighi del Ramadan. L'attentatore di Nizza era un uomo instabile psicologicamente, a causa della drammatica fase della sua vita, e privo di disciplina, perché non addestrato dall'IS, ai quali si sentiva legato soltanto da un punto di vista emozionale (Orsini, 2018).

È possibile affermare come una proporzione non trascurabile di jihadisti europei possieda trascorsi criminali e un'esperienza di detenzione in carcere. Tra i 125 foreign fighters "italiani" quasi la metà del totale dei jihadisti considerati risulta possedere precedenti penali prima della partenza per le zone di conflitto, cioè 55 casi, pari al 44\%. Il background criminale può essere caratterizzato da diversi reati, come per esempio vi possono essere soggetti già noti per azioni terroristiche ed eversive. Altre attività degne di essere menzionate sono la produzione e spaccio di sostanze stupefacenti, furto, rapina, ingresso e soggiorno illegale nel territorio nazionale e violenza privata. Invece, a livello europeo, i dati non appaiono omogenei, in quanto in alcuni paesi si contano un numero limitato di soggetti con trascorsi criminali ed in altri dove il fenomeno assume dimensioni più marcate. Se il fenomeno in Danimarca può essere comparabile a quello italiano, in Germania due terzi dei combattenti risulta aver avuto esperienze criminali pregresse, secondo le Autorità tedesche (Marone \& Vidino, 2018). 
Negli Stati Uniti il fenomeno risulta imponente e coinvolge quasi la totalità dei profili presi in considerazione, in quanto $1^{\prime} 85 \%$ dei terroristi, cioè 437 soggetti su 511, è risultato essere stato arrestato o incriminato per crimini ideologicamente motivati. Soltanto 57 jihadisti, pari all'11\%, sono apparsi non aver avuto la fedina penale "sporca" prima della radicalizzazione. La percentuale residua è da suddividere tra soggetti per cui mancano informazioni sulla variabile in esame, ovvero dodici, pari a circa il $2 \%$, e soltanto cinque per infrazioni minori, riconducibile a meno dell' $1 \%$ (START, 2018).

Per quanto concerne invece l'esperienza pregressa in carcere, analizzando gli attacchi di matrice jihadista eseguiti in Europa e Nord America tra il giugno 2014 e il giugno 2017, emerge come circa un terzo dei 65 attentatori, pari al 34\%, sia stato detenuto in carcere ad un certo punto della propria vita prima dell'attacco e come 22 attentati su 51 siano stati compiuti da individui che sono stati in carcere. La maggior parte di questi individui, tuttavia, non è stata detenuta per reati legati al terrorismo, bensì per questioni di droga, possesso di armi e violenza fisica, tra cui tentato omicidio, rapina e aggressione (Vidino, Marone \& Entenmann, 2017).

Le prigioni infatti possono essere luogo di radicalizzazione e reclutamento, consentendo ai soggetti già radicalizzati di stabilire o rafforzare connessioni reciproche, ed esponendo all'ideologia jihadista anche individui non ancora radicalizzati. Il fenomeno dei combattenti stranieri legati all'Italia ha visto 28 individui su 125 avere commesso reati tali da avere avuto una detenzione in carcere, per una percentuale contenuta che si aggira attorno al $22 \%$. Anche in questo caso, a livello europeo, i dati risultano eterogenei e si differenziano da paese a paese. In alcuni paesi il fenomeno risulta essere contenuto, mentre in altri la proporzione di soggetti con un passato da detenuti supera o si avvicina al 50\%, come il caso della Gran Bretagna, dove il 47\% dei combattenti convertiti aveva condanne alle spalle. La Francia risulta invece essere in linea con la media europea, pari al 33\% (Marone \& Vidino, 2018).

In conclusione, il jihadista è più frequentemente un soggetto maschio giovane, celibe e senza figli, con una condizione economica e un livello d'istruzione variabili, spesso con precedenti penali e dei trascorsi in carcere. Tra i tratti più rilevanti, vi è l'assenza di psicopatologie mentali rilevanti, in quanto terroristi si può diventare in seguito ad un'educazione molto rigorosa che porta a distinguere soltanto due categorie, il Bene e il Male. Il jihadista occidentale ha avuto dei traumi pregressi che lo hanno portato ad una condizione di marginalità sociale, primo step del processo della radicalizzazione.

Se le variabili sopra menzionate possono comparire più o meno frequentemente all'interno del profilo del jihadista, appurato l'elevato range di tratti psicologici, sociali, motivazioni e provenienze, è importante ricordare come il jihadista sia un fanatico. Pertanto, il fanatismo è quell'elemento che guida il comportamento del terrorista nelle sue azioni criminali in ogni loro aspetto (De Pasquali, 2016).

\section{Modus operandi}

In criminologia, per modus operandi si intendono tutte quelle azioni compiute dall'autore del reato e finalizzate alla commissione del reato stesso. Le modalità attraverso cui un gruppo terroristico realizza un attentato, creando così un proprio modus operandi, dipendono da molteplici fattori, come ad esempio la struttura del gruppo (Monzani, 2016).

Il modus operandi dei jihadisti è mutato nel tempo, a testimonianza di come il fenomeno jihadista sia un fenomeno dinamico, che si trasforma velocemente a seconda della realtà territoriale e del contesto storico e sociale. Con il declino di Arafat, con la caduta dell'Unione Sovietica e con la nascita di movimenti islamisti come Hamas e la Jihād Islamica Palestinese, il terrorismo palestinese, nato come una forma di terrorismo assai lontana dal fondamentalismo islamico, svolta verso il jihadismo e inizia ad incrementare l'utilizzo negli attentati dei kamikaze. Il kamikaze palestinese è generalmente un ragazzo giovane, con esperienze dirette di guerra e colpito dalla morte di familiari ed amici. Il suo status di uomo non sposato e il fatto di essere disoccupato, vengono sfruttati da organizzazioni terroristiche come Hamas, i quali promettono aiuti economici alle famiglie dei futuri martiri. Il martirio assume un carattere quasi sacrale a tal punto che le famiglie dei martiri che decidono di immolarsi per la causa palestinese esultano per la missione dei loro cari e non vedono il sacrificio come una tragedia. Al contrario, essi celebrano l'eroismo degli shahid, ottenendo inoltre aiuti sociali ed economici (De Pasquali, 2016).

Il terrorismo internazionale palestinese che tra la fine degli anni Sessanta e la fine degli anni Ottanta colpisce l'Europa, ritenuta complice di Israele, attacca con una frequenza impressionante, tanto che in quel periodo si contano 565 azioni terroristiche fuori dai confini israeliani che provocano 498 morti e 1.783 feriti. I target più colpiti dai terroristi palestinesi sono stati l'Italia con 64 episodi, seguita dalla Francia con 61, dalla Germania occidentale con 52, dalla Grecia con 35 e dalla Gran Bretagna con 32. L'azione terroristica palestinese coinvolse soprattutto mezzi di trasporto come gli aerei, permettendo così di colpire indiscriminatamente chiunque. L'attacco terroristico sicuramente più tragico e che torna immediatamente alla memoria è quello perpetrato il 5 settembre 1972, in occasione delle Olimpiadi di Monaco, quando otto terroristi di al-Fatah e Settembre Nero fanno irruzione negli appartamenti della delegazione israeliana uccidendo due atleti (Mastronardi \& Leo, 2005). Il più grande attentato di terrorismo internazionale, tuttavia, risulta essere, per la sua importanza da un punto di vista operativo, per la sua portata e per quello che ha rappresentato, l'attacco alle torri del World Trade Center a New York e al Pentagono di Washington 1'11 settembre 2001 per mano di gruppi legati ad al-Qaeda e Hamas. Per la prima volta il mondo ha assistito ad un'operazione su larga scala e realizzata da gruppi terroristici diversi collegati tra loro, segnando un punto di rottura con le tradizionali modalità di attacco terroristico suicida. Al fine di gestire un'azione di tali dimensioni e di garantire il successo dell'operazione, ai kamikaze è stato consegnato un vero e proprio manuale che doveva completare e supportare il loro addestramento psicologico e militare, riducendo al minimo le probabilità di ripensamenti da parte degli stessi terroristi (Mastronardi \& Leo, 2005).

Gli effetti drammatici dell'attacco sono stati conseguenza dell'imprevedibilità e della portata dell'attentato, nonché dalla sua natura "internazionale" e dalla novità che esso ha rappresentato (De Pasquali, 2016).

L'elemento che ricorre frequentemente nel modus operandi dei vari gruppi jihadisti è senza dubbio il suicidio religioso, modalità d'attacco che rappresenta il comune denominatore tra gruppi come i palestinesi, al-Qaeda e Daesh che presentano delle differenze, seppur condividendo l'ideologia del fondamentalismo religioso. Il terrorismo dell' 11 settembre si differenzia infatti notevolmente dal terrorismo odierno, sia per quanto riguarda l'organizzazione dei jihadisti e la pianificazione dell'attentato che, di conseguenza, per il modus operandi. Il terrorismo di al-Qaeda presenta quindi delle differenze con il terrorismo dell'IS (De Pasquali, 2016).

Al-Qaeda, nata come "la Base" in Afghanistan e come un gruppo terroristico centralizzato, durante il regime talebano, si propone l'obiettivo di combattere il nemico invasore straniero, individuato soprattutto negli Stati Uniti. Soltanto espellendo gli occidentali 
sarebbe stato possibile per Bin Laden ottenere i pieni poteri, fino ad allora escluso da un ruolo politico attivo. Il terrorismo di prima generazione di al-Qaeda è quindi il prodotto della scissione fenomenologica tra il fronte occidentale e i suoi oppositori, secondo il modello dei blocchi. Agli occhi degli occidentali la minaccia terroristica qaedista appare incomprensibile e indefinibile. Tuttavia, in seguito alla risposta americana agli attentati dell'11 settembre, il potenziale terroristico qaedista cala drasticamente, non rendendo più possibili attentati su larga scala come quelli realizzati all'inizio del nuovo millennio. Il nuovo leader al-Zawahiri dimostra di non possedere le stesse abilità del predecessore Bin Laden e la struttura centralizzata dell'organizzazione viene così smantellata. Avviene di conseguenza un cambiamento nelle logiche d'azione del gruppo, che agisce ora a livello regionale, senza obbedire ad una strategia coordinata ed organizzata. Al-Qaeda possiede da allora una struttura irregolare e fa ricorso, per i suoi attacchi, a tecniche di lotta di matrice terroristica. Questa tipologia di modus operandi non rientra invece nelle strategie dello Stato Islamico, nato ufficialmente per mano dell'auto-proclamato califfo al-Baghdadi, probabilmente la personalità più carismatica dell'intero panorama jihadista e con degli obiettivi ben chiari, ovvero la ricostruzione del Califfato riunendo le popolazioni sunnite. A differenza di al-Qaeda, l'IS rappresenta una vera e propria forza insurrezionale, che può contare su truppe di combattenti regolari che non ricorrono a tecniche di guerriglia ma a forme più tradizionali di scontro. Questa organizzazione possiede sin dall'inizio certamente più flessibilità tattica, ma il fatto di essere circoscritto entro definiti confini territoriali, più facilmente attaccabili, la rende maggiormente vulnerabile rispetto ad al-Qaeda, al contrario più decentrata e snella. Al-Qaeda si dimostra in questo senso più solida e compatta dal punto di vista tattico, contraddistinta da una suddivisione in cellule terroristiche, fattori che la rendono più difficile da estirpare definitivamente. Dall'altro lato, il gruppo di al-Baghdadi è sembrata possedere capacità militari e amministrative più elevate e maggiore dedizione e abilità nello sfruttamento dei canali mediatici. Tra le attività criminali più comuni che caratterizzano il modus operandi di questi gruppi jihadisti che permettono il finanziamento del gruppo stesso sono da segnalare i rapimenti e le uccisioni di ostaggi. Tuttavia, in questo contesto, queste attività vengono realizzate da al-Qaeda e dall'IS per differenti motivi. Mentre i militanti qaedisti rapiscono soprattutto per autofinanziarsi, Daesh vede in ciò anche un mezzo per diffondere globalmente il terrore tra i nemici (De Pasquali, 2016).

Mediaticamente scalpore, a tal proposito, ha creato l'episodio della giovane cooperante Silvia Romano, rapita dagli islamisti di al-Shabaab in Kenya il 20 novembre 2018 e rilasciata nel maggio 2020 dietro un presunto pagamento di un riscatto. La rilevanza mediatica di questa notizia è dovuta non solo alla rivelazione della conversione all'Islam della ragazza, che ora si fa chiamare Aisha, ma, dal punto di vista della sicurezza internazionale, ad un possibile aumento del rischio di rapimento per i cittadini italiani ed occidentali in zone controllate da organizzazioni come al-Qaeda e affiliati. Il rapimento a fine di riscatto infatti rappresenta una delle attività predilette dai gruppi terroristici come al-Qaeda per finanziarsi, i quali nel tempo sono diventati sempre più autosufficienti, utilizzando inoltre come fonte di finanziamento la tassazione o sistemi di estorsione nei territori controllati, contrabbando e narcotraffico. Originariamente, al contrario, al-Qaeda riceveva denaro su donazioni individuali, eventualmente redistribuendo fondi ai propri gruppi affiliati che ne avessero necessità (Sicuritalia, 2020).

Un'altra differenza riscontrabile tra al-Qaeda e l'IS è che quest'ultima da sempre ha preferito impiegare i propri militanti nella costruzione del Califfato in Siria e in Iraq, piuttosto che scagliarli contro le città occidentali. Secondo l'iniziale mentalità di Daesh, il tempo della propaganda jihadista di stampo qaedista, volta a scuo- tere le coscienze dei musulmani attraverso gli attentati contro l'Occidente, risulta scaduto e va sostituita con la costruzione di una realtà statuale che unisca tutti i fedeli musulmani (Orsini, 2016).

Mentre all'interno del mondo islamico il militante jihadista riesce ad esprimersi politicamente e militarmente, il nuovo terrorismo non può non considerare il destino di milioni di giovani musulmani che vivono nelle città europee, a maggioranza non musulmana, e che, in qualche modo, vengono affascinati dalla galassia jihadista. Questo musulmano è impossibilitato a combattere militarmente, ma ciò non esclude il perfezionamento delle proprie convinzioni religiose e, di conseguenza, il suo trasfigurarsi attraverso gesti estremi. Questa tendenza risponde alla logica "a ognuno il suo jihād" (Salvatori, 2018).

Come anche dimostrato dalle statistiche precedentemente riportate, il terrorista che agisce in Occidente è nella maggior parte dei casi un attentatore suicida "fai-da-te", un lupo solitario, un soggetto che agisce individualmente, in maniera solitaria, staccato da ogni organizzazione terroristica, che utilizza strumenti e mezzi improvvisati e che, soprattutto, si focalizza su target indifesi, di facile accesso e simbolicamente rilevanti, detti "soft target". Questi terroristi non hanno contatti con i membri del gruppo organizzato, se non per aver seguito un indottrinamento online, che li rende dei "self starters", ovvero individui musulmani che sono nati in Occidente che, però, sono impossibilitati a ricevere o rigettano ogni forma di integrazione culturale o sociale. Gli ordigni esplosivi utilizzati dal martire jihadista contemporaneo sono armi fabbricate seguendo istruzioni in rete e non provenienti direttamente dai mercati clandestini delle organizzazioni jihadiste. Il web viene inoltre utilizzato per lo scambio di informazioni tra estremisti, i quali apprendono e dispensano consigli su come scegliere gli obiettivi migliori da attaccare al fine di provocare un numero maggiore di vittime (De Pasquali, 2016). Il lupo solitario, rappresentante del nuovo terrorismo "molecolare" o "fluido", persegue così una linea d'azione indicatagli sul web, che gli lascia, tuttavia, discrezionalità sulle modalità e tempi dell'azione. Egli può quindi scagliarsi al grido di battaglia "Allah akbar" contro un obiettivo qualunque, individuato spesso tra gli infedeli, donando imprevedibilità all'attacco e maggiori possibilità di provocare numerose vittime. Anche in assenza di un elevato numero di mezzi a disposizione, il nuovo jihadista è disposto al sacrificio per la "giusta causa" contro il nemico vicino, rendendo il proprio gesto uno sprone per gli altri aspiranti combattenti. Al lupo solitario, tuttavia non viene preclusa alcuna possibilità. Qualora arrivasse la chiamata, il lupo solitario sarebbe disposto ad addestrarsi a diventare un combattente di Allah e a partire per rinforzare le fila dei foreign fighters, raggiungendo i compagni nei vari teatri regionali (Salvatori, 2018).

Il lupo solitario e il suo modus operandi possono essere considerati simili a quello del mass murder, che realizza le sue azioni in piena autonomia, senza però avere, a differenza del lupo solitario, alcuna affiliazione ideologica. Spesso si tende a pensare che le azioni dei lupi solitari siano il risultato di una propaganda tentacolare e ipnotica, capace di intercettare soggetti vulnerabili, indottrinarli e indurli così ad azioni efferate. In realtà la permeabilità dei lupi solitari all'ideologia jihadista non deriva da una propaganda straordinariamente persuasiva, ma da specifici tratti di personalità che, scompensati da eventi drammatici, spingono il soggetto alla ricerca di un'ideologia radicale. In un certo senso l'ideologia spinge i lupi solitari alle loro azioni, venendo perciò utilizzata pretestuosamente per giustificare azioni altrimenti folli (Piazza, 2019).

Secondo Orsini (2018), è possibile inquadrare il modus operandi di Daesh e dei "suoi" lupi solitari attraverso l'individuazione di tre categorie, che corrispondono a tre tipi diversi di attentato contro le città europee. Alla prima categoria appartengono quegli attentati decisi e organizzati dai vertici dell'IS, come ad esempio la 
strage di Parigi del 13 novembre 2015, quando nove jihadisti colpiscono il cuore della Francia e dell'Occidente utilizzando granate, mitragliatori e cinture esplosive e attaccando la città in sei punti diversi. Questo tipo di attentato è sicuramente il più pericoloso, in quanto meglio organizzato. I terroristi hanno contatti con altri jihadisti, armi, una forte motivazione psicologica che deriva dal fatto di godere della stima dei capi dell'IS, una maggiore disponibilità economica e di mezzi per portare a termine l'attacco e, di conseguenza, l'attentato risulta più devastante. Alla seconda categoria appartengono gli attentati dei lupi solitari, i quali possono essere distinti in addestrati e non addestrati, la maggioranza. Questa distinzione implica che questi attori sono imprevedibili e che, a differenza degli attentati della precedente categoria, tendono a causare un numero relativamente basso di vittime in quanto, a causa dell'impreparazione, commettono errori ingenui. L'unica eccezione è rappresentata dall'attacco di Nizza del 14 luglio 2016, quando Mohamed Bouhlel, durante la festa della presa della Bastiglia, si lancia con un camion contro la folla sulla Promenade, uccidendo 86 persone. A questa categoria appartengono inoltre quegli attentati analoghi a quello perpetrato il 22 marzo 2017 a Westminster, quando Khalid Masood, lupo solitario non addestrato, uccide quattro pedoni e un poliziotto con l'utilizzo di una vettura e di un coltello da cucina. I lupi solitari possono colpire qualunque città e con qualsiasi mezzo in quanto la loro finalità è esistenziale, nel senso che perseguono l'obiettivo di sentirsi parte di una comunità immaginata che dia un significato alla loro esistenza. Per questo motivo possono essere definiti dei "terroristi di vocazione", ossia coloro che cercano di appagare un bisogno interiore di riscatto e redenzione, ma che sono anche ossessionati dall'idea di punire il prossimo per la sua presunta corruzione morale. Alla terza categoria appartengono gli attentati delle microcellule autonome composte da jihadisti privi di addestramento e che non prendono ordini dai capi, come quelli di Barcellona e Cambrils in Spagna e del London Bridge. In Spagna sono coinvolti 12 terroristi, le cui azioni hanno provocato 14 vittime, un numero basso se si considera la proporzione tra numero di attentatori e di decessi. La proporzione appare decisamente diversa se confrontata con quella di Parigi, dove un commando di nove uomini ha colpito la città in sei punti diversi uccidendo 130 persone.

Un'altra interpretazione viene fornita da Alfredo Mantici, ex capo del dipartimento analisi del Sisde, che in un'intervista afferma come gli attentati jihadisti come quello di Molenbeeck e di Parigi siano molto simili a quelli dell'anarco-insurrezionalismo italiano ed europeo. Viene adottato un modello operativo che si basa sui cosiddetti "gruppi di affinità", ovvero tanto non organizzazioni piramidali dove si entra e poi non si esce più, ma piuttosto gruppi di amici, o addirittura parenti, che sulla base di una propaganda vista sul web si accordano per organizzare e compiere un attentato, per poi in seguito rientrare nel loro contesto sociale (Askanews, 2016).

Risulta ora necessario comprendere quale sia la logica con cui vengono scelti i bersagli che si differenziano in base alla natura del gruppo terroristico.

Nel contesto di interesse del lavoro, gli obiettivi maggiormente scelti sono i sopra citati "soft target", ovvero obiettivi di scarso valore ma simbolici e ad alto impatto psicologico che non richiedono una particolare pianificazione da parte dell'attentatore, né una particolare organizzazione logistica e una precisa attività di cellula. L'assaltatore, dunque, può pianificare, armarsi e agire da solo, senza avere nessun tipo di dipendenza da altri terroristi. Il "soft target", dunque, è un obiettivo ingaggiabile sia da un gruppo di terroristi che da un elemento che agisce al di fuori di qualsiasi organizzazione (Brasi \& Tatoni, 2020).

Secondo Orsini (2017) questa logica seguita dall'IS nella scel- ta dei target può essere riassunta nella frase "colpire coloro che ci colpiscono". Premesso che le risorse di Daesh sono ritenute scarse e che a causa di ciò è risultato impossibile per l'organizzazione jihadista realizzare un attentato sul modello di quello di Parigi, vertici del Califfato preferiscono investire le risorse a disposizione per colpire quei paesi occidentali responsabili degli attacchi in Medio Oriente alle basi jihadiste. In questo contesto, l'autore propone una gerarchia dell'odio jihadista, composta da quattro livelli. I paesi europei più odiati sono quelli che bombardano le roccaforti dell'IS in Siria e in Iraq, ovvero Francia, Inghilterra, Belgio, Olanda e Danimarca. Il secondo livello è rappresentato da quei paesi che inviano i militari a scontrarsi con i militanti dell'IS, cioè Turchia e Iran. Il terzo livello prevede quei paesi che si limitano a inviare i propri soldati a presidiare alcune strutture di pubblica utilità e ad addestrare i combattenti curdi. A questo livello appartiene 1'Italia, la quale mai ha, tuttavia, mosso concretamente guerra ai militanti dell'IS. L'ultimo livello è rappresentato da quei paesi inattivi, pur facendo parte della coalizione anti-IS.

Questa gerarchia sembra quindi spiegare il motivo per cui la Francia e l'Inghilterra sono risultati essere i paesi più colpiti dalla ferocia jihadista. Tuttavia, è possibile apparentemente muovere delle obiezioni, che tuttavia risultano non pertinenti. La prima è che la Germania è stata spesso colpita da attentati, pur non bombardando le postazioni islamiste. Eppure, è importante ricordare come il parlamento tedesco abbia ordinato in data 5 dicembre 2015 l'invio di mille militari tedeschi a supporto di quelli francesi, con l'aggiunta dell'impiego di una portaerei e alcuni aerei da ricognizione e da rifornimento che avevano il compito di assistere i caccia francesi. In seguito a quella data, i jihadisti cominciano quindi a mettere la Germania nel proprio mirino, attaccandola in diversi episodi. La seconda obiezione è che ci sono paesi europei che hanno subito attentati pur non facendo parte della gerarchia dell'odio jihadista, come la Spagna e la Svezia. La spiegazione di ciò è da ricercare nella classificazione stessa degli attacchi jihadisti, in quanto appare erroneo considerare tutti uguali gli attentati dell'IS. Gli attacchi a questi paesi sono infatti stati realizzati da lupi solitari e da microcellule autonome, che, come già visto, sono diversi dagli attentati pianificati dai capi. Essi possono colpire qualunque paese europeo, come dimostra il caso della Finlandia che, il 18 agosto 2017, è stata colpita da un lupo solitario marocchino al quale era stato negato l'asilo politico. Di conseguenza, se un lupo solitario colpisse un paese come l'Italia, l'IS rivendicherebbe, ma ciò non significa automaticamente che l'attentato sia stato pianificato dai vertici dell'organizzazione (Orsini, 2017).

Un'altra interessante osservazione, utile a comprendere il modus operandi di una organizzazione come l'IS, è che, come dimostra l'esperienza di Boko Haram in Nigeria e al-Shabaab in Somalia, che lottano per instaurare il Califfato nei loro territori, il numero degli attentati terroristici all'estero aumentano quando i militanti jihadisti perdono terreno nei loro territori. Volendo fornire un esempio, la strage dell'Università di Garissa, in Kenya, del 2 aprile 2015 è stata conseguenza dell'arretramento di al-Shabaab verso le periferie della Somalia, non potendo più dedicarsi in questo modo alla costruzione del Califfato. Analogamente, Boko Haram ha iniziato a realizzare numerosi attentati terroristici a seguito del duro attacco del Camerun, Ciad, Niger e Nigeria, paesi che sono finanziati dagli Stati Uniti per liberare la Nigeria dal terrorismo islamista. Anche in questo caso, nel momento in cui Boko Haram è stata costretta a interrompere l'edificazione del Califfato in Nigeria, ha iniziato a realizzare una grande quantità di attacchi terroristici all'estero. Da questi esempi è possibile asserire che se la costruzione della società jihadista viene interrotta da interventismi nemici, i terroristi tentano di distruggere la società di coloro che ne hanno provocato l'arresto. Tutto ciò accade perché i jihadisti, dotati di razionalità e di intenzio- 
nalità, odiano coloro da cui sono attaccati o da cui si sentono attaccati. Questa regola, valida sia per al-Qaeda che per lo Stato Islamico, fa comprendere ancora una volta come i jihadisti agiscano come qualunque altra persona nella società, in collera verso coloro che gli hanno provocato un torto. (Orsini, 2016).

L'ultimo elemento fondamentale del modus operandi delle organizzazioni jihadiste è rappresentato dalla rivendicazione, in cui le organizzazioni terroristiche delineano gli scopi dell'attentato, le finalità e richieste del gruppo terroristico. Per quanto riguarda gruppi terroristici come al-Qaeda e Daesh, la rivendicazione rappresenta uno strumento fondamentale per la diffusione del terrore e per la presentazione dei gruppi stessi come gli autori di un attentato. In questo senso Daesh, grazie alla sua grande capacità di gestione dei canali di informazione, spesso rivendica non solo attentati pianificati e realizzati dai vertici dell'organizzazione, ma anche azioni terroristiche perpetrate da individui non strettamente collegati ad essa come i lupi solitari. È questo il caso della maggior parte degli attentati commessi in Europa negli ultimi anni, attacchi sfruttati da al-Baghdadi per rafforzare l'immagine del gruppo terroristico agli occhi degli occidentali, diffondendo il terrore, e dei simpatizzanti e potenziali nuovi militanti (Salvatori, 2018).

La causa di ciò è riconducibile al fenomeno durkheimiano dell'"effervescenza collettiva", molto alta quando un'organizzazione terroristica riscontra successo. L'effervescenza collettiva è una delle teorizzazioni del sociologo francese Emile Durkheim e con essa si intende quel fenomeno che si viene a creare nel momento in cui gli individui creano un nuovo ordine sociale e lo fanno vivendo un'esperienza collettiva che ai loro occhi appare segnata dalla straordinarietà (Pace, 2007). In particolare, "quando un'organizzazione terroristica raccoglie successi eclatanti, $i$ suoi simpatizzanti si esaltano e ricevono una forte spinta emozionale a lanciarsi nel conflitto. Quando, invece, un'organizzazione jihadista precipita nella polvere, l'effervescenza collettiva si riduce e $i$ suoi simpatizzanti si deprimono" (Orsini, 2017, pag. 15).

La spettacolarizzazione mediatica degli attentati e l'ingresso dell'IS nelle case degli occidentali attraverso le immagini hanno favorito almeno inizialmente i processi di radicalizzazione proprio perché hanno contribuito ad accrescere il clima di effervescenza collettiva in favore dell'organizzazione. Per questo, occorrerebbe difendersi dalla disinformazione attraverso lo studio accademico, unico strumento in grado di smascherare i fenomeni di manipolazione dei media (Orsini, 2018).

\section{Bibliografia}

Beaumont P., (2016), Mohamed Lahouaeij-Bouhlel: Who Was the Bastille Day Truck Attacker? The Guardian.

Bergema R., Van Sam M., (2018), Waves of the black banner: An Exploratory Study on the Dutch Jihadist Foreign Fighters in Syria and Iraq, Studies in Conflict \& Terrorism.

De Luca R., (2002), Il terrore in casa nostra. Nuovi scenari per il terrorismo globale del XXI secolo, Franco Angeli, Milano.

De Luca R., Macrì C., Zoli B., (2013), Anatomia del crimine in Italia. Manuale di criminologia, Giuffrè Editore, Milano.

De Pasquali P., (2016), Criminologia transculturale ed Etnopsichiatria forense. Terrorismo, immigrazione, reati culturalmente motivati, Alpes Italia, Roma.

Galimberti U., (2006), Dizionario di psicologia, UTET, Torino.

Heinke D.H., (2017), German Foreign Fighters in Syria and Iraq: The updated Data and Its Implications, CTC Sentinel, vol.10, n.3.

Hogg M.A., Vaughan, G.M., (2012), Psicologia sociale. Teorie e applicazioni, Pearson Italia, Milano-Torino.
Hole G., (1995), Fanatismo. La propensione all'estremismo e le sue radici psichiche, San Paolo, Milano.

Horgan J., (2015), Psicologia del terrorismo, Edra, Milano.

Lombardi M., (2016), Il terrorismo nel nuovo millennio, Vita e Pensiero, Milano.

Mastronardi V.M., Leo S., (2005), Terroristi, Newton \& Compton Editori, Roma.

Monzani M., (2016), Manuale di criminologia, Libreriauniversitaria. it Edizioni, Padova.

Orsini A., (2016), Isis. I terroristi più fortunati del mondo e tutto ciò che è stato fatto per favorirli, Rizzoli, Milano.

Orsini A., (2017), I tre tipi di attentati dell'Isis che spiegano perché quanto l'Italia è in pericolo, Il Foglio, 29 agosto.

Orsini A., (2018), L'Isis non è morto. Ha solo cambiato pelle, Rizzoli, Milano.

Orsini A., (2019), Il terrorismo in Africa, Luiss University Press, Roma.

Pace E., (2007), Introduzione alla sociologia delle religioni, Carocci Editore, Roma.

Palermo G.B., Mastronardi V.M., (2005), Il profilo criminologico. Dalla scena del crimine ai profili socio-psicologici, Giuffrè Editore, Milano.

Pellicani L., (2002), Dalla società chiusa alla società aperta, Rubbettino, Soveria Mannelli.

Picozzi M., (2016), Profiler, Sperling \& Kupfer, Milano.

Salvatori P., (2018), Spie? L'intelligence nel sistema di sicurezza internazionale, La Lepre Edizioni, Roma.

Sbailò C., (2014), Diritto pubblico dell'Islam mediterraneo, Linee evolutive degli ordinamenti nordafricani contemporanei: Marocco, Algeria, Tunisia, Libia, Egitto, Cedam, Milano.

Silber M., (2012), The al Qaeda factor. Plots against the West, University of Pennsylvania ress, Philandelphia.

Sinclair A., (2003), Storia del terrorismo, Newton \& Compton Editori, Roma.

Teti A., (2018), Cyber Espionage e cyber counterintelligence. Spionaggio e controspionaggio cibernetico, Rubbettino Editore, Soveria Mannelli.

Vidino L., Marone F., Entenmann E., (2017), Jihadista della porta accanto. Radicalizzazione e attacchi jihadisti in Occidente, Ledizioni, Milano.

Vidino L., Marone F., (2018), Destinazione jihad. I foreign fighters d'Italia, Ledizioni, Milano.

Zoja L., (2017), Nella mente di un terrorista. Conversazione con Omar Bellicini, Giulio Einaudi Editore, Torino

\section{Sitografia}

Alexander A., (2016), Cruel Intentions: Female Jihadists in America, Program on Extremisms, The George Washington University, Washington. https://extremism.gwu.edu/sites/g/files/zaxdzs 2191/f/downloads/Female\%20Jihadists\%20in\%20America.pdf

Askanews (2016), Terrorismo, Mantici (ex Sisde): noi più preparati per prevenzione. https:/www.askanews.it/video/2016/04/28/terrorismo-mantici-ex-sisde-noi-pi\%c3\%b9-preparati-per-prevenzione-20160428_video_14384122//

Brasi C., Tatoni G., (2020), La trasformazione del ihad in Europa. Profili psicologici e dottrinali, 21 giugno, Analytica for intelligence and security studies. https:/www.analyticaintelligenceandsecurity.it/ricerca-e-analisi/la-trasformazione-della-jihadin-europa-profili-psicologici-e-dottrinali/

Cadalanu G., (2020), Coronavirus, per l'Isis è una punizione agli infedeli, 28 marzo, La Repubblica. https://www.repubblica. 
it/esteri/2020/03/28/news/coronavirus per 1 isis e una punizione agli infedeli-252504501/

Consiglio dell'Unione Europea (2005). Strategia antiterrorismo dell'Unione Europea, 30 novembre, Bruxelles. https://register. consilium.europa.eu/doc/srv? $=\mathrm{IT} \& \mathrm{f}=\mathrm{ST} \% 2014469 \% 202005 \%$ 20REV\%204

Europol (2020), EU terrorism situation \& trend report 2020, European Union Agency for Law Enforcement Cooperation. https://www.europol.europa.eu/activities-services/mainreports/terrorism-situation-and-trend-report-2019-te-sat

La Repubblica.it (2019), Militari italiani feriti in Iraq, Isis rivendica l'attacco: "Feriti quattro crociati e quattro apostati", 11 novembre. https://www.repubblica.it/esteri/2019/11/11/news/attentato_iraq_militari_italiani_feriti_di_maio-240829562/
Limes Online (2015), Chi sono e da dove vengono i foreign fighters. https://www.limesonline.com/chi-sono-e-da-dove-vengono-iforeign-fighters/76298

National Consortium for the Study of Terrorism and Responses to Terrorism. START (2018), Profiles of Individual Radicalization in the United States - PIRUS (Keshif) .https://www.start.umd. edu/profiles-individual-radicalization-united-states-pirus-keshif

Piazza S. (2019), Lupi solitari. I percorsi della radicalizzazione e le strategie di contrasto, 25 febbraio, Babilon. https://www.babilonmagazine.it/lupi-solitari-i-percorsi-della-radicalizzazione-ele-strategie-di-contrasto/

Sicuritalia (2020), Al-Qaeda e il rischio di sequestri, 15 maggio. https://www.sicuritalia.it/news/risk-security-solutions/al-quaeda-e-il-rischio-di-sequestri/
Correspondence: Lorenzo Ortenzi.

E-mail: 1rz.ortenzi@libero.it

Key words: jihadist; terrorist cause; mechanisms of adhesion. Parole chiave: jihadista; causa terrorista; meccanismi di adesione. Palabras clave: yihadista; causa terrorista; mecanismos de adhesión.

Received for publication: 9 June 2020.

Accepted for publication: 2 July 2020.

This article is distributed under the terms of the Creative Commons Attribution Noncommercial License (by-nc 4.0) which permits any noncommercial use, distribution, and reproduction in any medium, provided the original author(s) and source are credited.

${ }^{\circ}$ Copyright: the Author(s), 2020

Licensee PAGEPress, Italy

Rivista di Psicopatologia Forense, Medicina Legale, Criminologia 2020; $25: 549$

doi:10.4081/psyco.2020.549 